NBER WORKING PAPER SERIES

\title{
THE TIME-SERIES PROPERTIES OF AGGREGATE CONSUMPTION: IMPLICATIONS FOR THE COSTS OF FLUCTUATIONS
}

\author{
Ricardo Reis \\ Working Paper 11297 \\ http://www.nber.org/papers/w11297 \\ NATIONAL BUREAU OF ECONOMIC RESEARCH \\ 1050 Massachusetts Avenue \\ Cambridge, MA 02138 \\ April 2005
}

I am grateful to Gadi Barlevy, Per Krusell, Yves Nosbusch, Jonathan Parker, Chris Sims, Lars Svensson, and Mark Watson for useful comments. Contact: rreis@ princeton.edu. First draft: November 2004. The views expressed herein are those of the author(s) and do not necessarily reflect the views of the National Bureau of Economic Research.

(C2005 by Ricardo Reis. All rights reserved. Short sections of text, not to exceed two paragraphs, may be quoted without explicit permission provided that full credit, including $\odot$ notice, is given to the source. 
The Time-Series Properties of Aggregate Consumption: Implications for the Costs of Fluctuations Ricardo Reis

NBER Working Paper No. 11297

April 2005

JEL No. E32, E21, E60

\begin{abstract}
While this is typically ignored, the properties of the stochastic process followed by aggregate consumption a.ect the estimates of the costs of fluctuations. This paper pursues two approaches to modelling aggregate consumption dynamics and to measuring how much society dislikes fluctuations, one statistical and one economic. The statistical approach estimates the properties of consumption and calculates the cost of having consumption fluctuating around its mean growth. The paper finds that the persistence of consumption is a crucial determinant of these costs and that the high persistence in the data severely distorts conventional measures. It shows how to compute valid estimates and confidence intervals. The economic approach uses a calibrated model of optimal consumption and measures the costs of eliminating income shocks. This uncovers a further cost of uncertainty, through its impact on precautionary savings and investment. The two approaches lead to costs of fluctuations that are higher than the common wisdom, between $0.5 \%$ and $5 \%$ of per capita consumption.

Ricardo Reis

Princeton University

Department of Economics

324 Bendheim Hall

Princeton, NJ 08544-1021

and NBER

rreis@princeton.edu
\end{abstract}




\section{Introduction}

In a famous contribution, Robert Lucas Jr. (1987) asked: what would be the effect on welfare of eliminating economic fluctuations? As Lucas (1987, page 3) put it, answering this question would allow us "to get a quantitative idea of the importance of stabilization policy relative to other economic questions." To reach an answer, Lucas made three assumptions. First, he assumed that society's preferences can be represented by a welfare function that depends on the time path of consumption per capita alone. That is, he assumed not only that there is a representative consumer, but also that her utility function represents society's normative preferences. Second, he assumed that this welfare function is time-separable and iso-elastic. Third, he assumed that the log of annual per capita consumption is serially uncorrelated and normally distributed around a linear trend. These three assumptions produced a surprising result: society would be willing to sacrifice a meagre $0.05 \%$ of consumption to get rid of fluctuations. The economic fluctuations that macroeconomists have focused so much attention on cost each person on average only $\$ 12$ per year.

A large literature has followed focusing especially on the first two assumptions. Imrohoroğlu (1989), Atkeson and Phelan (1994), Krusell and Smith (1999), Storesletten, Telmer and Yaron (2001), Beaudry and Pages (2001), and Krebs (2003, 2004) measured the costs of fluctuations in economies where agents are heterogeneous and markets are incomplete, so that there is not a representative consumer whose preferences are a valid measure of welfare. While it is conceivable that the costs of fluctuations would be higher, as bad income shocks hurt a few households severely, the typical finding from these studies is that the costs of fluctuations are only slightly higher or even lower than the Lucas benchmark. Other studies looked at the second assumption of iso-elastic preferences. Dolmas (1998), Otrok (1999), Tallarini (2000), and Epaulard and Pommeret (2003) assumed different utility functions, while Alvarez and Jermann (2004) used asset prices to elicit rather than assume preferences over risk. While many of these studies found much larger estimates of the costs of fluctuations, this came typically at the expense of assuming people are extremely averse to risk, which appears to be inconsistent with the risk-taking that we observe in their choices (Lucas, 2003).

The focus of this paper is on the third assumption that consumption is serially uncorrelated. I will present alternative models of consumption dynamics and study their impact 
on estimates of the costs of fluctuations. I will consider statistical models of aggregate consumption and show that if consumption is very persistent, as is the case in the U.S. data, Lucas' (1987) estimates are severely downward-biased. A methodological contribution of this paper is to show how to construct reliable estimates of the costs of fluctuations when there is persistence of the degree that we observe.

Together with statistical models, I will also consider economic models in which consumption fluctuations are an optimal response to shocks. One virtue of having endogenous consumption choices is that it uncovers a cost of fluctuations that is typically ignored in the literature: the existence or not of fluctuations affects the level and growth rate of consumption by affecting the desire for precautionary savings and for risky investment. The discipline imposed across the different models is that they must all match the main features of the aggregate consumption data.

The Lucas assumption that shocks to consumption are serially uncorrelated is clearly dismissed by the data. More surprisingly, either interpreted through an economic model or using an estimated statistical model, the adequate process for aggregate consumption implies that the costs of fluctuations are actually one or two orders of magnitude than Lucas argued. The estimates in this paper suggest that the costs of fluctuations are between $0.5 \%$ and $5 \%$ of per capita consumption.

It is important to be clear about how these estimates should be used. This paper measures the costs of eliminating the uncertainty that makes consumption fluctuate. These numbers do not distinguish between fluctuations due to productivity or monetary shocks, or between those that correspond to business cycles and those that are due to uncertainty about long-run growth. In terms of economic theories, what these large numbers suggest is that focusing attention on deterministic growth models, as it happened at least partly in response to Lucas' original results, will be missing out on a significant part of welfare. Section 6 of this paper will discuss how the results in this can be used to assess the costs associated with business cycles more specifically.

Even though the assumption that consumption is serially uncorrelated is clearly at odds with the data, it has received little attention in the literature. A few studies have modelled consumption instead as a random walk (Dolmas, 1998, Tallarini, 1999, and Epaulard and Pommeret, 2003), but their focus was on the other assumptions behind the Lucas' calculations. This paper instead systematically investigates the effect of the stochastic properties 
of the consumption data on the costs of fluctuations. This focus leads the paper to address some problems with making inferences about the costs of fluctuations, which the literature has so far ignored. More related to this paper is Obstfeld (1995), who found that modeling consumption as a random walk only slightly increased the costs of fluctuations. I will show that this conclusion hinges on the way in which Obstfeld calibrated the parameters of his model; an alternative approach, which is more in accord with the data, gives the opposite result. On the side of theory, this paper shares with Barlevy (2004) the emphasis on measuring the costs of fluctuations without excluding the possibility that these may have long-lasting effects, either through long-lived fluctuations or through an impact on the average growth rate.

The paper proceeds as follows. Section 2 presents some simple models of consumption that highlight the main determinants of the costs of fluctuations. These involve choosing some key parameters, and section 3 discusses the evidence that will guide the choice of values for these parameters. Section 4 estimates the costs of fluctuations across a variety of statistical models for consumption, while section 5 uses instead economic models. Section 6 concludes by interpreting the economic significance of the estimates.

\section{Models of consumption and the costs of fluctuations}

A central tenant of most theories of choice under uncertainty is that people dislike risk. If society faced a choice between its current risky consumption series $\left\{C_{t}\right\}$ and a "suitably modified" consumption series $\left\{\bar{C}_{t}\right\}$ that is purged from fluctuations, it is assumed that society would choose the latter. As Lucas (1987) emphasized, it is important to go one step further and be able to quantify this preference for stability. He suggested measuring the costs of fluctuations by the fraction of annual consumption that society would be willing to pay to eliminate these fluctuations. Maintaining his assumptions of a utility function that is time-separable (with discount rate $\rho$ ) and iso-elastic (with a coefficient of relative risk aversion $\gamma$ ), the costs of fluctuations $(\lambda)$ are defined as the solution to: ${ }^{1}$

$$
E\left[\sum_{t=0}^{\infty} e^{-\rho t}\left(\frac{\left(C_{t}(1+\lambda)\right)^{1-\gamma}-1}{1-\gamma}\right)\right]=\sum_{t=0}^{\infty} e^{-\rho t}\left(\frac{\bar{C}_{t}^{1-\gamma}-1}{1-\gamma}\right)
$$

\footnotetext{
${ }^{1} E[$.$] denotes the expectation operator conditional on information at time 0$.
} 
Solving this equation requires two pieces of information. First, one needs the stochastic process for the risky consumption path in order to evaluate the expectation. Second, one must define precisely what the counterfactual "suitably modified" consumption series is. Both of these requirements are met by having a model for consumption. This paper will consider two distinct approaches to modelling consumption: one consists of estimating a statistical process for consumption; the other consists of assuming an economic environment in which society optimally chooses how much to consume.

\section{Statistical models of consumption}

From a statistical perspective, a natural choice for the counterfactual consumption series is expected consumption. The exercise of eliminating fluctuations then corresponds to eliminating the variability of consumption, while keeping its mean unchanged. One of the stylized facts about economic growth in the United States in the past century is that consumption, like income, has grown at an approximately constant rate. An appropriate model for counterfactual consumption is: $\bar{C}_{t}=E\left[C_{t}\right]=C_{0} e^{g t}$.

I will maintain the assumption that consumption is log-normally distributed. The U.S. data is consistent with this assumption and it is analytically convenient since it leads to the following simple expressions for the costs of fluctuations: ${ }^{2}$

$$
\ln (1+\lambda)=\left\{\begin{array}{l}
0.5\left(1-e^{g-r}\right) \sum_{t=0}^{\infty} e^{(g-r) t} \operatorname{Var}\left(c_{t}\right) \quad \text { if } \gamma=1 \\
\frac{1}{\gamma-1} \ln \left[\left(1-e^{g-r}\right) \sum_{t=0}^{\infty} e^{(g-r) t} e^{0.5 \gamma(\gamma-1) \operatorname{Var}\left(c_{t}\right)}\right] \quad \text { if } \gamma \neq 1
\end{array}\right.
$$

Small letters denote the natural logarithm of the respective capital letter, e.g., $c_{t}=\ln \left(C_{t}\right)$. In the expressions, I replaced the (unobservable) discount rate by the (observable) average real interest rate $r$, using Ramsey's result that with iso-elastic preferences, $\gamma g \cong r-\rho$.

Estimating the costs of fluctuations now requires only calculating the forecast error variance of consumption at different horizons. This, in turn, requires a model of consumption dynamics. One simple model of de-trended consumption is

$$
c_{t}=\eta c_{t-1}+\varepsilon_{t}
$$

where $\varepsilon_{t}$ is normally distributed with mean zero and variance $\sigma^{2}$. This representation fits

\footnotetext{
${ }^{2}$ The calculations leading to this and most other results are in the appendix.
} 
the post-war U.S. consumption data well: lagged consumption can account for $84 \%$ of the variability of present consumption when $\eta$ equals the least squares estimate 0.92 . Moreover, special cases of (3) correspond to two important processes. Lucas (1987) assumed that $\eta=0$ and I will correspondingly call this the Lucas consumption process. Hall (1978) showed that optimally chosen consumption dynamics approximately follow a random walk and that the U.S. data is consistent with this assumption. This corresponds to the case $\eta=1$, which I will label the Hall consumption process.

With this $\mathrm{AR}(1)$ model and if $|\eta| \leq 1$, the costs of fluctuations approximately equal:

$$
\begin{aligned}
\lambda & \cong \frac{0.5 \gamma \sigma^{2}}{r-g+1-\eta^{2}} \\
& =\frac{0.5 \gamma\left(1-\eta^{2}\right)}{r-g+1-\eta^{2}} \times\left(\frac{\sigma^{2}}{1-\eta^{2}}\right) .
\end{aligned}
$$

These formulae shows clearly the role of different parameters on the costs of fluctuations. The roles of $\gamma$ and $r-g$ and their calibration will be discussed in section 3. The focus of this paper is on the properties of the stochastic process for consumption on the costs of fluctuations. In this case, these are captured by the two parameters $\sigma^{2}$ and $\eta$.

The first expression (4) shows that $\lambda$ increases with both the variability and the persistence of consumption. The larger is the variability of shocks to consumption, the more society finds these shocks costly, so the more it is willing to pay to eliminate consumption fluctuations. The more persistent are shocks to consumption, the more long-lived is their impact on consumption, and thus the larger their cost. ${ }^{3}$ Still, for $r-g=0.02$, which section 3 will justify, even when $\eta$ is as high as 0.8 so that a shock to consumption takes about two years to dissipate by half, the costs of fluctuations are only twice higher than those with a process with no persistence. As persistence increases further though, the costs of fluctuations increase quite rapidly. If $\eta$ is 0.9 , the costs are already 7 times larger than with a Lucas process, and if $\eta=0.95$ they are 14 times higher. The impact of the persistence on the costs of fluctuations is more dramatic when we shift from the Lucas to the Hall models. If $r-g=0.02$, then the Hall consumption model predicts costs of fluctuations that are 51

\footnotetext{
${ }^{3}$ When $\rho=0$, the formula in (4) differs from the one derived by Lucas (1978) by a factor of $1 /(1+r-g)$. This difference arises because I evaluate expected utility conditional on information at time 0 , whereas Lucas computes the unconditional expectation. Since $r-g$ is close to zero, this difference is quantitatively negligible. I focus on the conditional rather than the unconditional expectations, since in the latter case the costs of fluctuations would be infinite when $\rho=1$ and would be severely downward biased when $\rho$ is close to 1 since the unconditional variance would be estimated using the relatively short post-war U.S. sample.
} 
times larger than those estimated by Lucas. If $r-g=0.01$, another value that section 3 will show is consistent with the data, the costs of fluctuations are two orders of magnitude larger than what Lucas estimated.

These calculations assumed that $\sigma^{2}$ was held fixed while $\eta$ varied. It might be argued that Lucas (1987) instead measured the unconditional variance of consumption, which corresponds to $\sigma^{2} /\left(1-\eta^{2}\right)$. In expression (5), the first term actually decreases as $\eta$ rises. The reason is that keeping the unconditional variance fixed, raising $\eta$ increases the predictability of consumption by lowering its forecast error variance. The consumer therefore faces less risk so the costs of fluctuations fall. However, rather than undermining the argument of the previous paragraph, instead this alternative view of the Lucas (1987) calculation provides an alternative demonstration of its limitations. Lucas (1987) used a finite sample to gauge the unconditional variance of consumption. This implies that if consumption is very persistent, his estimate is severely downward biased. This is particularly clear in the case where consumption follows a random walk: while in a finite sample one obtains a finite estimate of the variance of consumption, the actual variance is infinite. Even if consumption is stationary, if it is very persistent, one will obtain a very downward-biased estimate of its variance using the post-war U.S. sample.

Whichever way you look at it, what these calculations show is that it is crucial to jointly estimate both the volatility of shocks to consumption and their persistence. One needs a statistical model for consumption to calculate the costs of fluctuations. Section 4 will attack this estimation problem directly using different statistical approaches.

\section{Economic models of consumption}

An economic model of consumption starts with a specification of the environment facing a representative consumer earning a random income stream. ${ }^{4}$ The consumption process is then whatever is optimally chosen. The counterfactual consumption with no fluctuations is what the consumer would choose if income was stable.

\footnotetext{
${ }^{4}$ To focus solely on the third of the Lucas (1987) assumptions, I will maintain the assumption of a representative consumer. It would be interesting in future work to both model the consumption process carefully and to take into account the large idiosyncratic risks facing households (Parker and Preston, 2004).
} 
In this section, I consider a simple economic environment. The consumer solves:

$$
\begin{gathered}
\max _{\left\{C_{t}\right\}} E\left[\sum_{t=0}^{\infty} e^{-\rho t}\left(\frac{C_{t}^{1-\gamma}-1}{1-\gamma}\right)\right] \\
\text { s.t.: } K_{t+1}+C_{t}=R_{t} K_{t} .
\end{gathered}
$$

The budget constraint states that savings $\left(K_{t+1}\right)$ plus consumption equals income. Last period's savings are the only source of income through investment in a risky technology with positive marginal return $R_{t}$, which is log-normally distributed with mean $r-0.5 \sigma^{2}$ and variance $\sigma^{2}$. The consumer starts at time 0 with some positive amount of capital $K_{0}$.

The appendix shows that the solution to this problem is:

$$
\begin{gathered}
c_{t}=c_{t-1}+g-0.5 \sigma^{2}+\varepsilon_{t}, \\
\text { where } g=(r-\rho) / \gamma+0.5(\gamma+1) \sigma^{2}-\sigma^{2}, \\
\text { with initial condition } C_{0}=\left(1-e^{g-r}\right) R_{0} K_{0}
\end{gathered}
$$

In this model, consumption follows a random walk as in the Hall statistical model. However, there is one important difference between the two models. Now, both the level and the growth rate of consumption are functions of $\sigma^{2}$. Income uncertainty not only causes fluctuations in consumption but also has two effects on the level and growth rate of consumption, captured by the two terms on the right-hand side of the expression for $g$. The first effect is due to precautionary savings: the rational consumer reacts to the uncertainty by saving more. This allows her to accumulates a stock of precautionary savings to safeguard against unexpected future bad shocks. The second effect is due to investment risk: the risk-averse consumer will shy away from the investing in the risky technology. In this model, as long as relative risk aversion exceeds one, the combined precautionary-investment effect is such that eliminating fluctuations would raise the level of consumption and reduce growth. ${ }^{5}$

The counterfactual $\bar{C}$ therefore differs from average consumption both in the level of initial consumption and in its growth rate. While one can follow Lucas and calculate the gains from eliminating fluctuations in consumption, one needs a theory of consumption choices to calculate the costs of fluctuations in income. The latter affect not just the

\footnotetext{
${ }^{5}$ Barlevy (2004) has suggested a complementary channel through which fluctuations affect growth. Eliminating uncertainty may raise investment in innovative activities and consequently long-run growth.
} 
fluctuations in consumption, but also the level and growth rate of consumption through the precautionary-investment motive.

Moreover, note that this precautionary-investment effect is more general than the model in this section. It will be present in most economic models of consumption under uncertainty, regardless of their predictions for the persistence of consumption. ${ }^{6}$ Likewise, while growth may be higher or lower without uncertainty, welfare will always be higher. By ignoring this effect, statistical models will necessarily underestimate the costs of fluctuations.

\section{Initial estimates of the costs of fluctuations}

Table 1 presents estimates of the costs of fluctuations for the different models that I have discussed so far. The value of $\sigma^{2}$ for each model is estimated using U.S. annual data from 1947 to 2003 on real per capita consumption of non-durables and services from the Bureau of Economic Analysis. This will be the measure of consumption used in this paper. Quarterly data leads to very similar results; total consumption, which inappropriately includes expenditure on durables as current consumption, approximately doubles the estimate of $\sigma^{2}$ and so doubles all of the estimates of the costs of fluctuations. As for the choice of values for $\gamma$ and $r-g$, it will be discussed at length in section 3 .

Panel A displays the estimates with the Lucas model of consumption. As Lucas (1987) originally concluded, fluctuations cost very little, between $0.04 \%$ and $0.2 \%$ of per capita consumption. Panel B presents estimates for the AR(1) statistical model fitted to the U.S. data. The estimated $\eta$ implies a considerable amount of persistence, with a half-life of deviations from trend growth after a shock of 8 years. However, the estimated costs still lie in the same range as the Lucas estimates. ${ }^{7}$ These results should be interpreted with great care though; section 4 will show that these estimates are statistically inconsistent and severely downward biased.

Panel C shifts to the economic model presented in this section. The infinite persistence of shocks and the precautionary savings effect combine to generate substantially larger costs of fluctuations, between $0.2 \%$ and $3.1 \%$. This upper bound is almost 80 times larger than

\footnotetext{
${ }^{6}$ Epaulard and Pommeret (2003) find an effect of volatility on growth in an AK-growth model, but interpret it as being specific to endogenous growth models. Actually, this effect is present in most models of consumption and uncertainty.

${ }^{7}$ The reader may be surprised that the estimates in panel B are actually lower than those in panel A, in spite of the higher persistence. The reason is that the estimated volatility of shocks is lower for the $\operatorname{AR}(1)$ than for the Lucas model, which drives down the costs of fluctuations.
} 
the smallest number in Panel A that Lucas focused on.

Table 1 - Estimates of the costs of fluctuations in three simple models

\begin{tabular}{|c|c|c|c|}
\hline \multicolumn{4}{|c|}{ Panel A: The Lucas statistical model } \\
\hline & $\gamma=1$ & $\gamma=3$ & $\gamma=5$ \\
\hline & $0.04 \%$ & $0.12 \%$ & $0.20 \%$ \\
\hline & $(\$ 9)$ & $(\$ 28)$ & $(\$ 46)$ \\
\hline \multicolumn{4}{|c|}{ Panel B: The AR(1) statistical model estimated by least squares } \\
\hline & $\gamma=1$ & $\gamma=3$ & $\gamma=5$ \\
\hline \multirow{2}{*}{$r-g=0.03$} & $0.03 \%$ & $0.10 \%$ & $0.17 \%$ \\
\hline & $(\$ 8)$ & $(\$ 24)$ & $(\$ 40)$ \\
\hline \multirow{2}{*}{$r-g=0.02$} & $0.04 \%$ & $0.11 \%$ & $0.18 \%$ \\
\hline & $(\$ 8)$ & $(\$ 25)$ & $(\$ 43)$ \\
\hline \multirow{2}{*}{$r-g=0.01$} & $0.04 \%$ & $0.12 \%$ & $0.19 \%$ \\
\hline & $(\$ 9)$ & $(\$ 27)$ & $(\$ 45)$ \\
\hline
\end{tabular}

Panel C: The random walk economic model

\begin{tabular}{clll} 
& $\gamma=1$ & $\gamma=3$ & $\gamma=5$ \\
$r-g=0.03$ & $0.21 \%$ & $0.62 \%$ & $1.03 \%$ \\
& $(\$ 48)$ & $(\$ 145)$ & $(\$ 242)$ \\
$r-g=0.02$ & $0.31 \%$ & $0.94 \%$ & $1.56 \%$ \\
& $(\$ 73)$ & $(\$ 219)$ & $(\$ 365)$ \\
$r-g=0.01$ & $0.63 \%$ & $1.88 \%$ & $3.14 \%$ \\
& $(\$ 147)$ & $(\$ 441)$ & $(\$ 735)$ \\
\hline \hline \multicolumn{2}{l}{ Each cell shows the per capita costs of fluctuations as a fraction of consumption } \\
and, in brackets, in 2003 dollars. The standard deviation of shocks is 0.028, 0.011, \\
and 0.011, for panels A to C respectively.
\end{tabular}

After a brief detour in the next section to discuss the calibration of $\gamma$ and $r-g$, the remainder of this paper explores more refined estimates of the costs of fluctuations. Section 4 estimates statistical models that deal with the high persistence of consumption data, while section 5 builds more elaborate economic models of consumption. To preview the results, most models will suggest that the costs of fluctuations are closer to those in Panel C.

\section{Choice of parameters}

Risk aversion 
The extent to which society dislikes risk is measured by the coefficient of relative risk aversion $\gamma$. While this parameter is at the center of many economic models, there is some disagreement on its value. The wider disagreement comes from looking at either data on consumption choices, or data on financial market prices.

Data on consumption choices suggests a value for relative risk aversion between 1 and 5. Arrow (1971) originally made a case for relative risk aversion equal to 1 on theoretical grounds. Friend and Blume (1975) looked at variation in portfolio allocations between households and found values between 2 and 4 . The consumption of leisure can also be used to elicit preferences towards risk. Chetty (2005) shows that the choices by consumers facing the risk of unemployment combined with plausible values for the income and wage elasticities of labor supply imply that the coefficient of relative risk aversion is at most 2 . People's choice of careers with risky income profiles also runs against very high risk aversion (Saks and Shore, 2004), as does their choice to hold most of their wealth in illiquid housing that carries a significant amount of risk (Cocco, 2003).

Starting with Mehra and Prescott (1985), economists have realized that financial market prices imply much higher risk aversion. In order to account for the large premium that equity pays over Treasury bills requires that relative risk aversion is at least 50 and close to 200. This paper offers no solution to this disparity between consumption and financial price data. The approach taken here (as the title indicates) is to use consumption data to measure the costs of fluctuations, so I will use values for relative risk aversion between 1 and 5. The hope is that these measurements are more robust to advances in the theory linking consumption choices to financial markets. ${ }^{8}$

\section{Discounting future costs}

The difference between the return on savings and the growth rate of consumption, $r-g$, equals the growth rate of the marginal utility of consumption. This is the effective rate at which consumers discount the impact of shocks on future consumption. The smaller is $r-g$, the less people discount the future costs of a shock that persists for at least a few periods. Thus, the larger the overall costs of fluctuations.

\footnotetext{
${ }^{8} \mathrm{~A}$ few recent papers offer some hints at reconciling a reasonable degree of risk aversion with asset prices using precisely observations on consumption. Gabaix and Laibson (2003) and Chetty and Szeidl (2004) show that the infrequent adjustment of plans regarding total or parts of consumption, due to either inattentive behavior or consumption commitments, can explain the equity premium.
} 
The average return on savings and the average consumption growth rate are both observable in the data, so calibrating $r-g$ is a relatively easy matter. Poterba (1998) estimates the after-tax return on capital in the United States in the period 1959-1996 to be either $3.9 \%$ or $5 \%$, depending on whether one includes property taxes or not. McGrattan and Prescott (2003) use data from 1880 to 2002 and find returns of $4 \%$ on accounting capital, and $5.4 \%$ on equity. As for the average annual growth rate of per capita consumption, it equals $2.2 \%$. These point estimates therefore suggest a value for $r-g$ somewhere between $1.7 \%$ and $2.2 \%$. Correspondingly, I will consider the values of $1 \%, 2 \%$, and $3 \%$ for $r-g$.

\section{The role of the intertemporal elasticity of substitution}

Obstfeld (1995) argued that it is important to distinguish between risk aversion and intertemporal substitution when calculating the costs of fluctuations. He noted that while risk aversion determines the per-period cost of volatility, intertemporal substitution determines the weights given to the future cumulative per-period costs.

To investigate this claim, I consider the specification of preferences due to Epstein and Zin (1989) and Weil (1990). Utility at time t, $V_{t}$, is defined by the recursion:

$$
\left[1+\left(1-e^{-\rho}\right)(1-\gamma) V_{t}\right]^{(1-\theta) /(1-\gamma)}=\left(1-e^{-\rho}\right) C_{t}^{1-\theta}+e^{-\rho}\left[1+\left(1-e^{-\rho}\right)(1-\gamma) E_{t}\left[V_{t+1}\right]\right]^{(1-\theta) /(1-\gamma)}
$$

The parameter $\gamma$ still equals the coefficient of relative risk aversion. Nevertheless, now the intertemporal elasticity of substitution equals $1 / \theta$. With the expected utility preferences in (8), the elasticity of intertemporal substitution equals the inverse of relative risk aversion, so the two concepts cannot be distinguished. (You can see this by noting that if $\gamma=\theta$, then (9) becomes (6).)

Solving for optimal consumption and for the costs of fluctuations in the economic model in (9) and (7) is an easy matter. The appendix contains the calculations, which lead to the following surprising result: The costs of fluctuations with Epstein-Zin-Weil preferences (9) are the same as the costs with iso-elastic preferences (6) up to a term in $O\left(\sigma^{4}\right)$. Therefore, distinguishing between intertemporal substitution and risk aversion does not affect the estimates of the costs of fluctuations. Moreover, the intertemporal elasticity of substitution does not enter the formula for the costs of fluctuations.

How can this finding be reconciled with Obstfeld's (1995) conclusion? The explanation 
lies in the following expression that holds with the preferences in (9):

$$
r=\rho+\theta g-0.5 \gamma \sigma^{2}(\theta-1)
$$

This paper used the available direct observations on $r$, the parameter that directly affects the costs of fluctuations. Obstfeld (1995) instead chose a value for $\rho$. Therefore, his choice of the elasticity of intertemporal substitution affected the value attributed to the return on capital via expression (10), which in turn affected the estimates of the costs of fluctuations. Because Obstfeld (1995) set $\rho$ at 0.05, and $\theta$ between 2 and 20, he implicitly attributed a value for the average after-tax return to capital between $9 \%$ and $49 \%$ per annum. Thus, his calculations heavily discounted the future costs arising from persistent shocks, which explains why he found that going from the Lucas to the Hall consumption models had little effect on the costs of fluctuations.

\section{Statistical models of consumption}

\section{Which process for consumption? Lucas versus Hall}

Section 2 showed that the Lucas and Hall statistical models of consumption imply very different costs of fluctuations. Because these models impose a rigid structure on the timeseries of consumption, one can test which best describes the data.

Table 2 shows the results from different tests of the null hypothesis that consumption has a unit root: the original (augmented) test of Dickey and Fuller (1979), the alternative due to Phillips and Perron (1988), the point-optimal test of Elliott, Rothenberg and Stock (1996), and finally the modified Phillips-Perron $\left(\mathrm{MZ}_{\mathrm{t}}\right)$, point-optimal $\left(\mathrm{MP}_{\mathrm{t}}\right)$, and Barghava statistic (MSB) tests combined with a modified Schwarz criteria to select the lag length. These last three test were suggested by $\mathrm{Ng}$ and Perron (2001) in order to account for size distortions if the underlying data process is stationary. The results are clear: the null hypothesis corresponding to the Hall process is never rejected at the $5 \%$ significance level.

The last row of the table presents the result of a test, by Kwiatkowski et al (1992), of the null hypothesis that consumption is trend stationary. The data rejects this hypothesis at the $5 \%$ significance level. 
Table 2 - Statistical tests of whether consumption has a unit root

\begin{tabular}{|c|c|c|c|}
\hline Test & Test statistic & $5 \%$ critical value & Decision \\
\hline \multicolumn{4}{|l|}{ Null hypothesis: Unit Root } \\
\hline Dickey-Fuller & -1.88 & -3.49 & not rejected \\
\hline Phillips-Perron & -1.74 & -3.49 & not rejected \\
\hline Elliott-Rothenberg-Stock & 10.95 & 5.71 & not rejected \\
\hline \multicolumn{4}{|l|}{ Ng-Perron: } \\
\hline $\mathrm{MZ}_{\mathrm{t}}$ & -2.02 & -2.91 & not rejected \\
\hline MSB & 0.24 & 0.17 & not rejected \\
\hline $\mathrm{MP}_{\mathrm{t}}$ & 10.83 & 5.48 & not rejected \\
\hline \multicolumn{4}{|l|}{ Null hypothesis: Stationarity } \\
\hline Kwiatkowski et al & 0.17 & 0.15 & rejected \\
\hline
\end{tabular}

There is a simple way to understand why the data clearly favors the Hall process over the Lucas process. It is possible to nest the two models in a single regression equation:

$$
c_{t}-c_{t-1}=\text { const. }+u_{t}-\beta u_{t-1},
$$

where $u_{t}$ is the residual. The Lucas process imposes the restriction $\beta=1$, while the Hall process requires that $\beta=0$.

The 1947-2003 U.S. data produces an estimate of $\beta$ of -0.36 with a standard error of 0.13. Not only is the estimate lower than one, it is not even positive - thus the strong statistical rejection of the Lucas model. However, note that while the Hall model is closer to the data, it is also rejected at the $5 \%$ significance level. Consumption growth is positively serially correlated, a fact that has inspired most modern research on consumption. ${ }^{9}$ Fitting the facts requires richer models of consumption dynamics; the rest of this section investigates different possibilities.

\footnotetext{
${ }^{9}$ See Fuhrer (2000) and Reis (2004) for two alternative models that try to account for this positive serial correlation, either by appealing to habits or to costs of processing information.
} 


\section{Estimating the persistence of U.S. consumption}

A statistical model for consumption that is more general than either the Lucas or the Hall models is the $\mathrm{AR}(1)$ in (3), where $\eta$ is not restricted to necessarily equal either one or zero. A naive application of this model would be to estimate $\eta$ by least squares and, if this estimate is below 1, apply the formula in (4). This was the procedure that led to the estimates in panel B of table 1.

However, it is well-understood that for very persistent series like consumption, the leastsquares estimate of $\eta$ is downward-biased. For example, if the true model is a random walk, then the least squares estimate of $\eta$ will be below 1 with a probability of $68 \%$. Given how steeply costs increase with $\eta$ when it is close to 1 , this can lead to severely under-estimating the costs of fluctuations.

The most popular way to deal with this problem is to model $\eta$ as lying within a circle of radius $c / n$ around 1 , where $n$ is the size of the available sample. The estimate of the new parameter $c$ (a "Pitman" drift) has a distribution that can be characterized using local-tounity asymptotics (Stock, 1994). Since deterministic formulae link $c$ to $\eta$ and in turn to $\lambda$, this characterizes the distribution of the estimate of the costs of fluctuations.

In the data, the confidence intervals for $\eta$ include a large region well above one. The formula in (2) would then imply that the costs of fluctuations are estimated to be infinity with a probability of more than $30 \%$. This result arises because forecast error variances far ahead shoot quickly to infinity. This highlights one weakness of directly applying the formula in (2) if consumption follows an explosive process. The estimate of the costs of fluctuations in this case is dominated by estimates of the variability of consumption at horizons very far ahead, well above the size of the finite sample in which they were estimated.

The local-to-unity model suggests a natural way to deal with this issue. That model assumes that as the sample size increases, consumption becomes closer to a random walk; likewise, one can calculate the costs of fluctuations assuming that after the sample horizon, the forecast error variance is indistinguishable from that of a random walk. Focusing for now on the case of log utility, one estimator that formalizes this suggestion is

$$
\hat{L}=0.5\left(1-e^{g-r}\right)\left[\sum_{t=0}^{n} e^{(g-r) t} \hat{v}\left(c_{t}\right)+\sum_{t=n+1}^{\infty} e^{(g-r) t}\left[\hat{v}\left(c_{n}\right)+\hat{v}\left(c_{1}\right)(t-n)\right]\right]
$$

where $\hat{v}\left(c_{t}\right)$ is the least squares estimator of the the forecast error variance $t$ steps ahead. 
This estimator replaces $\hat{v}\left(c_{t}\right)$ for horizons that exceed the size of the sample, by the $n^{t h}$ step-ahead forecast error variance for a random walk. ${ }^{10}$ As $n \rightarrow \infty$, this estimator coincides with the exact value the costs of fluctuations: $\hat{L} \rightarrow \ln (1+\lambda)$. In a finite sample, under the maintained local-to-unity model, this is the estimator that is within $1 / n$ of the costs of fluctuations. ${ }^{11}$

In the $\mathrm{AR}(1)$ model, straightforward but tedious algebra shows that, using the approximation $1+c / n=\exp (c / n)+O\left(1 / n^{2}\right)$ :

$$
\hat{L}=0.5 \hat{\sigma}^{2} e^{g-r}\left[\sum_{t=0}^{n} e^{(g-r+2 c / n) t}+e^{(g-r) n} /\left(1-e^{g-r}\right)\right]
$$

where $\hat{\sigma}$ is the least-squares estimate of the standard error of shocks. It is simple to show that as $n \rightarrow \infty, \hat{\sigma}^{2} \rightarrow \sigma^{2}$. Applying the functional central limit theorem:

$$
\frac{1}{n} \sum_{t=0}^{n} e^{(g-r-2 c / n) t} \Rightarrow \int_{0}^{1} e^{(g-r+2 U) s} d s
$$

where $\Rightarrow$ denotes weak convergence. $U$ equals the random variable $\left(\int_{0}^{1} J(s) d W(s)\right) /\left(\int_{0}^{1} J(s)^{2} d s\right)$, where $J($.$) is an Orstein-Uhlenbeck process d J(s)=c J(s) d s+d W(s)$ and $W($.$) is a standard$ Brownian motion. The continuous mapping theorem then implies that:

$$
\frac{\hat{L}}{n} \Rightarrow 0.5 \sigma^{2} e^{g-r} \frac{e^{g-r+2 U}-1}{g-r+2 U}
$$

which fully describes the asymptotic distribution of the estimate of the costs of fluctuations.

According to this asymptotic result, the least squares estimate of the costs of fluctuations is not only an inconsistent estimate of the true costs, but moreover, it converges to a random variable. The reason is that as $n$ grows, the least squares estimation errors persist for longer rather than dying off. This implies that the estimates in panel B of table 1 were downward-biased. Yet using the formula in (15), constructing median-unbiased estimates and confidence intervals for the costs of fluctuations is possible. ${ }^{12}$

\footnotetext{
${ }^{10}$ For a random walk, $\operatorname{Var}\left(c_{t}\right)=\sigma^{2} t$, so $\operatorname{Var}\left(c_{t}\right)=\operatorname{Var}\left(c_{n}\right)+\operatorname{Var}\left(c_{1}\right)(t-n)$.

${ }^{11}$ This approach has a close relative in Phillips's (1998) construction of confidence intervals for far-ahead impulse responses in the local-to-unity model.

${ }^{12}$ The distribution of $U$ is not only non-normal but it also depends on the (unknown) value of $c$. It therefore requires many numerical simulations to characterize this distribution for each value of $c$. Stock (1991) has already done the work of tabulating the distribution of $U$. Since $\hat{L} / n$ increases monotonically with $U$, one use his tables to construct confidence intervals for the estimates of the costs of fluctuations.
} 
In the appendix, I extend the calculations in this section in two directions. First, I consider the case when relative risk aversion is different from one. Second, I extend the statistical model to the Dickey-Fuller regression form:

$$
\Delta c_{t}=\kappa_{0}+\kappa_{1} t+\varrho c_{t-1}+\sum_{j=1}^{k} \psi_{j} \Delta c_{t-j}+u_{t} .
$$

Now, it is the largest autoregressive root that is modelled as $1+c / n$. This allows for a more flexible characterization of log consumption, as a $k+1^{\text {th }}$ order autoregressive process with a drift and a time trend.

Table 3 presents median-unbiased estimates and $90 \%$ confidence intervals for the estimated costs of fluctuations if consumption dynamics are described by (16). The costs are now much higher than the naive estimates in table 1 . They range from $0.2 \%$ to $3.2 \%$ of per capita consumption and even the lower bounds of the confidence intervals are higher than those in the panel B of table 1. According to these calculations, society substantially dislikes the current variability in consumption.

Table 3 - The costs of fluctuations when consumption is persistent

\begin{tabular}{cccc}
\hline \hline \multirow{2}{*}{ Panel A: Costs in percentages of annual per capita consumption } \\
& $\gamma=1$ & $\gamma=3$ & $\gamma=5$ \\
$r-g=0.03$ & $0.21 \%$ & $0.63 \%$ & $1.05 \%$ \\
& $(0.19 ; 0.21)$ & $(0.57 ; 0.64)$ & $(0.95 ; 1.07)$ \\
$r-g=0.02$ & $0.31 \%$ & $0.95 \%$ & $1.58 \%$ \\
& $(0.29 ; 0.32)$ & $(0.87 ; 0.96)$ & $(1.46 ; 1.61)$ \\
$r-g=0.01$ & $0.63 \%$ & $1.90 \%$ & $3.19 \%$ \\
& $(0.60 ; 0.64)$ & $(1.81 ; 1.92)$ & $(3.03 ; 3.22)$ \\
\hline
\end{tabular}

Panel B: Costs in annual per capita 2003 dollars

\begin{tabular}{cccc} 
& $\gamma=1$ & $\gamma=3$ & $\gamma=5$ \\
$r-g=0.03$ & $\$ 49$ & $\$ 147$ & $\$ 246$ \\
& $(44 ; 50)$ & $(133 ; 150)$ & $(223 ; 256)$ \\
$r-g=0.02$ & $\$ 74$ & $\$ 222$ & $\$ 371$ \\
& $(68 ; 75)$ & $(204 ; 225)$ & $(341 ; 377)$ \\
$r-g=0.01$ & $\$ 148$ & $\$ 446$ & $\$ 747$ \\
& $(140 ; 149)$ & $(423 ; 450)$ & $(709 ; 755)$ \\
\hline \hline
\end{tabular}

Each cell shows the median unbiased estimate and, in parenthesis, the $90 \%$ confidence interval. The Ng and Perron (2001) modified BIC picked the autoregression's order. 


\section{Parametric unrestricted estimates}

The evidence at the beginning of this section strongly suggested that consumption is not stationary. The data does not reject the null hypothesis that the first difference of consumption is stationary however. (The unit root tests are not reported here for brevity.) Wold's theorem states that any stationary series has a moving average representation. A general statistical model for consumption then is

$$
\Delta c_{t}=\text { const. }+A(L) u_{t}
$$

where $\Delta c_{t}=(1-L) c_{t}$ and $A(L)=\sum_{i=0}^{\infty} a_{i} L^{i}$, and $L$ is the lag operator $L^{i} u_{t}=u_{t-i}$.

If consumption follows this process, the costs of fluctuations in (2) become:

$$
\ln (1+\lambda)=\left\{\begin{array}{lc}
0.5 \sigma^{2}\left(1-e^{g-r}\right)\left(\sum_{t=1}^{\infty} e^{(g-r) t} \sum_{j=0}^{t-1} \sum_{i=0}^{j} a_{i}^{2}\right) \quad \text { if } \gamma=1 \\
\frac{1}{\gamma-1} \ln \left[\left(1-e^{g-r}\right)\left(1+\sum_{t=1}^{\infty} e^{(g-r) t} e^{0.5 \sigma^{2} \gamma(\gamma-1) \sum_{j=0}^{t-1} \sum_{i=0}^{j} a_{i}^{2}}\right)\right] & \text { if } \gamma \neq 1
\end{array}\right.
$$

It is impossible to estimate the infinite number of parameters $a_{i}$ with a finite number of observations. However, it has long been known that an ARMA model

$$
B(L) \Delta c_{t}=\text { const. }+C(L) \varepsilon_{t},
$$

where $B(L)$ and $C(L)$ are lag polynomials of low order, typically provides a good approximation to the dynamics of most macroeconomic series. Given estimates of the ARMA model, one can easily recover the parameters $a_{i}$ using the relation $A(L)=B(L)^{-1} C(L)$.

Estimating (19) requires choosing the order of $B(L)$ and $C(L)$. I restricted the range of admissible models to a maximum of $3 \mathrm{AR}$ and/or MA parameters. ARMA processes with many parameters are notoriously difficult to estimate and the experience with ARMA modelling has been that low-order ARMA processes typically have a superior forecasting performance. I estimated the 16 admissible models by maximum likelihood. ${ }^{13}$ To pick between them, I used the Bayesian information criterion (BIC). This criterion picks the model with the highest likelihood, while imposing a penalty that increases with the number

\footnotetext{
${ }^{13}$ One important concern with estimating ARMA models is that the likelihood functions are often multipeaked or nearly flat for a wide range of parameter values, so numerical procedures can converge on incorrect estimates. To safeguard against this possibility, I plotted the likelihood functions, examined their gradients at the proposed optima, and started the numerical maximizations from different initial values.
} 
of parameters being estimated. One advantage of the BIC is that, as the sample size goes to infinity, it consistently picks the true underlying model. The BIC picked the $\operatorname{ARMA}(2,2)$ as the best model, followed by the $\operatorname{ARMA}(1,0)$ and by the $\operatorname{ARMA}(0,1)$.

\section{Table 4 - Estimates of the costs of fluctuations from ARMA models}

\begin{tabular}{cccc}
\hline \hline Panel A: Estimated ARMA & $(2,2)$ model & \\
$\left(1-0.66 L-0.32 L^{2}\right) \Delta c_{t}=\left(1+1.03 L+0.56 L^{2}\right) u_{t}$, & $\sigma_{u}=0.011$ \\
& $\gamma=1$ & $\gamma=3$ & $\gamma=5$ \\
$r-g=0.03$ & $0.31 \%$ & $0.94 \%$ & $1.60 \%$ \\
$r-g=0.02$ & $(\$ 72)$ & $(\$ 219)$ & $(\$ 375)$ \\
& $0.47 \%$ & $1.43 \%$ & $2.47 \%$ \\
$r-g=0.01$ & $(\$ 109)$ & $(\$ 334)$ & $(\$ 579)$ \\
& $0.94 \%$ & $2.93 \%$ & $5.33 \%$ \\
& $(\$ 220)$ & $(\$ 687)$ & $(\$ 1248)$ \\
\hline
\end{tabular}

Panel B: Estimated ARMA $(1,0)$ model

\begin{tabular}{cccc}
$\quad(1-0.34 L) \Delta c_{t}=u_{t}$, & $\sigma_{u}=0.010$ & \\
& $\gamma=1$ & $\gamma=3$ & $\gamma=5$ \\
$r-g=0.03$ & $0.40 \%$ & $1.23 \%$ & $2.13 \%$ \\
$r-g=0.02$ & $(\$ 94)$ & $(\$ 288)$ & $(\$ 498)$ \\
& $0.61 \%$ & $1.89 \%$ & $3.33 \%$ \\
$r-g=0.01$ & $(\$ 144)$ & $(\$ 442)$ & $(\$ 780)$ \\
& $1.25 \%$ & $3.94 \%$ & $7.40 \%$ \\
\hline
\end{tabular}

Panel C: Estimated ARMA $(0,1)$ model

\begin{tabular}{cccc}
$\Delta c_{t}=(1+0.36 L) u_{t}$, & $\sigma_{u}=0.011$ & \\
& $\gamma=1$ & $\gamma=3$ & $\gamma=5$ \\
$r-g=0.03$ & $0.34 \%$ & $1.02 \%$ & $1.76 \%$ \\
$r-g=0.02$ & $(\$ 79)$ & $(\$ 240)$ & $(\$ 412)$ \\
& $0.51 \%$ & $1.56 \%$ & $2.73 \%$ \\
$r-g=0.01$ & $(\$ 120)$ & $(\$ 366)$ & $(\$ 638)$ \\
& $1.03 \%$ & $3.23 \%$ & $5.92 \%$ \\
\hline \hline
\end{tabular}

Each cell shows the per capita costs of fluctuations as a fraction of consumption and, in brackets, in 2003 dollars.

Table 4 shows the costs of eliminating fluctuations in consumption for these three statistical models. The first conclusion to take from the table is that the estimates are all larger than the corresponding estimates in Panel $\mathrm{C}$ of table 1. The positive serial correlation in consumption growth implies that shocks propagate by more over time than what 
the Hall model predicted. A second conclusion is that across the three empirical consumption processes, the estimates of the costs of fluctuations are roughly similar. The results are robust in the sense that moving between models that fit the data almost equally well does not drastically affect the estimates. This leads to the third conclusion: the costs of fluctuations are approximately between $0.5 \%$ and $5 \%$ of per capita consumption, similar to the estimates in table 3 .

\section{Non-parametric unrestricted estimates}

The key empirical inputs into the formula for the costs of fluctuations in (2) are the forecast error variances of consumption. So far, I have estimated these by fitting parametric models to the observations of consumption. A natural alternative is to estimate the forecast error variances directly imposing as little structure as possible on the model of consumption.

Since these variances are conditional on information at time zero, then $\operatorname{Var}\left(c_{t}\right)=$ $\operatorname{Var}\left(c_{t}-c_{0}\right)$. It is difficult to estimate these without specifying what the conditioning information at time 0 is. However, doing so is close to specifying a parametric model for consumption, precisely what this section is trying to avoid. I overcome this dilemma by estimating the unconditional variance of the $t^{t h}$ difference in log consumption. The conditional and unconditional variances will be the same in the case of the $\operatorname{AR}(1)$; otherwise, the unconditional variance will be higher. The estimates in this section therefore provide non-parametric upper bounds on the costs of fluctuations.

Cochrane (1987) showed that the unconditional variance of the $t^{t h}$ difference in consumption equals:

$$
t \sigma_{\Delta c}^{2}\left(1+2 \sum_{j=1}^{t-1} \frac{t-j}{t} R_{j}\right)
$$

$R_{j}$ is the $j^{\text {th }}$ order autocorrelation of the first difference of consumption; $\sigma_{\Delta c}^{2}$ is its variance. The quantity in parenthesis is the Bartlett estimator of the spectrum of the first-difference of consumption at frequency zero using a lag window of length $t$. The sample autocorrelations and the sample variance of the first difference of consumption provide consistent estimates of these moments, so it is an easy mater to evaluate this expression. ${ }^{14}$

One difficulty is that it is impossible to compute the variance of the $t^{\text {th }}$ difference in

\footnotetext{
${ }^{14}$ I multiply the expression in parenthesis by $n /(n-t+1)$ to improve the performance of the estimator in a small sample (Cochrane, 1987).
} 
consumption if $t$ is larger than the sample size. Even if $t$ is smaller than $n$, as long as it is close to it, the estimator of $R_{t}$ will be using only a few observations. I tackle this problem in the same way that I did earlier when deriving the asymptotic distribution of the costs of fluctuations. I use an estimator like $\hat{L}$ in (12), with the only difference that the first sum now includes terms only up to a fraction of $n$. This way, the estimator only requires computing the variances of consumption differences up to a fraction of the sample. As before, this estimator asymptotically converges to the true costs of fluctuations and it provides a good approximation in a finite sample if consumption is very persistent.

Table 5 - Estimates of costs of fluctuations from variance estimates

\begin{tabular}{cccc}
\hline \hline Panel A: Estimating correlations of order up to $25 \%$ of the sample \\
& $\gamma=1$ & $\gamma=3$ & $\gamma=5$ \\
$r-g=0.03$ & $0.25 \%$ & $0.76 \%$ & $1.29 \%$ \\
& $(\$ 59)$ & $(\$ 179)$ & $(\$ 303)$ \\
$r-g=0.02$ & $0.36 \%$ & $1.10 \%$ & $1.87 \%$ \\
& $(\$ 85)$ & $(\$ 257)$ & $(\$ 440)$ \\
$r-g=0.01$ & $0.68 \%$ & $2.10 \%$ & $3.68 \%$ \\
& $(\$ 160)$ & $(\$ 492)$ & $(\$ 863)$ \\
\hline
\end{tabular}

Panel B: Estimating correlations of order up to $50 \%$ of the sample

$\begin{array}{lccc} & \gamma=1 & \gamma=3 & \gamma=5 \\ r-g=0.03 & 0.26 \% & 0.78 \% & 1.33 \% \\ r-g=0.02 & (\$ 61) & (\$ 183) & (\$ 311) \\ & 0.37 \% & 1.12 \% & 1.91 \% \\ r-g=0.01 & (\$ 86) & (\$ 262) & (\$ 447) \\ & 0.69 \% & 2.12 \% & 3.71 \% \\ & (\$ 161) & (\$ 496) & (\$ 871)\end{array}$

Panel C: Estimating correlations of order up to $75 \%$ of the sample

$\begin{array}{llll} & \gamma=1 & \gamma=3 & \gamma=5 \\ r-g=0.03 & 0.16 \% & 0.48 \% & 0.81 \% \\ r-g=0.02 & (\$ 37) & (\$ 113) & (\$ 189) \\ & 0.22 \% & 0.68 \% & 1.15 \% \\ r-g=0.01 & (\$ 52) & (\$ 159) & (\$ 271) \\ & 0.48 \% & 1.47 \% & 2.60 \% \\ & (\$ 112) & (\$ 346) & (\$ 610)\end{array}$

Each cell shows the per capita costs of fluctuations as a fraction of consumption and, in brackets, in 2003 dollars.

Table 5 contains the new estimates of the costs of fluctuations. From panels A to C, I 
use increasing fractions of the sample, from $25 \%$ to $50 \%$ to $75 \%$. The costs of fluctuations from using this approach are typically in between the random walk estimates and the larger estimates using ARMA models. They are all larger than the Lucas benchmark of $0.05 \%$.

\section{Economic models of consumption}

The components of the model

One pervasive model of consumption and fluctuations is the neoclassical stochastic growth model, in which a representative consumer solves:

$$
\begin{array}{ll} 
& \max _{\left\{C_{t}\right\}} E\left[\sum_{t=0}^{\infty} e^{-\rho t}\left(\frac{C_{t}^{1-\gamma}-1}{1-\gamma}\right)\right] \\
\text { s.t.: } \quad & K_{t+1}=A_{t}^{1-\alpha} K_{t}^{\alpha}+(1-\delta) K_{t}-C_{t} .
\end{array}
$$

The new notation refers to: $A_{t}$ - stochastic productivity, $\alpha$ - the capital share, and $\delta$ - the depreciation rate. ${ }^{15}$

I set the depreciation rate at 0.05 , the value typically chosen in the literature; close alternatives do not greatly affect the estimates of the costs of fluctuations. Choosing the process for productivity and the value of the capital share requires more attention though. These affect a key determinant of the costs of fluctuations: the persistence of consumption.

All else equal, the faster diminishing returns set in, the more transient the effect of shocks to consumption. With a Cobb-Douglas production function, the capital share determines the speed of convergence. The assumption in the model of section 2 was that there were constant returns to savings. Considering the broad investment possibilities available to society, this might just be the right assumption (Knight, 1944). Constant returns also imply that shocks have a permanent effect. Thus, there are no consumption dynamics associated with the transition to a steady state. Some of the endogenous growth literature claims that this may be an appropriate approximation of reality; for instance, King and Rebelo (1993) argued that transitional dynamics likely are quantitatively insignificant.

\footnotetext{
${ }^{15}$ Otrok (2001) also uses an estimated business cycle model to investigate the costs of fluctuations. However, his model has many other features (habits, two sectors of production, etc.) and his approach to calculating the welfare cost of fluctuations is different and does not capture the precautionary-investment effect that I emphasize.
} 
Barro and Sala-i-Martin (2003) instead pointed to the evidence of conditional convergence of income levels across countries as supporting transitional dynamics and diminishing returns to capital accumulation. As they discuss at length, the existing estimates of the speed of convergence point to a value of the capital share around 0.75 . This is consistent with a broad vision of capital that includes both physical and human capital. If $K_{t}$ stands solely for physical capital though, the U.S. data on factor payments suggest instead that the capital share is about 0.36 . Having considered the case $\alpha=1$ in section 2 , I now examine these two alternatives.

The second factor driving the persistence of consumption is the process driving productivity shocks. I will model productivity as:

$$
a_{t}=\mu+\tau(1-\phi) t+\phi a_{t-1}+w_{t}, \text { with } w_{t} \sim N\left(0, \omega^{2}\right)
$$

Prescott (1986) discusses how this process provides a good approximation to the observations of the Solow residual. While these observations imply that productivity is highly persistent, it is difficult to distinguish in the data between a stationary process, with say $\phi=0.9$, or a non-stationary process, in which case $\phi=1$. Moreover, Kydland and Prescott (1982) found that a real business cycle model performs equally well with either option. I will consider both cases.

Aside from persistence, the other key determinant of the costs of fluctuations is the volatility of consumption. In this economic model, consumption volatility is driven by the parameter $\omega$. It is difficult to pinpoint this value in the data, since the Solow residual is likely a very noisy measure of productivity. Consistent with this paper's overall approach, I calibrate this parameter to match the properties of consumption. Namely, I set $\omega$ so that the model matches either the standard deviation of log consumption (for the stationary model), or the standard deviation of its first difference (for the non-stationary model).

\section{Solving for the costs of fluctuations}

I solve the four models corresponding to the different assumptions on $\alpha$ and $\phi$ by loglinearizing around the non-stochastic steady state. Unfortunately, when productivity is nonstationary, I am only able to solve the model when $\gamma=1$. With non-stationary productivity, the model does not have a steady state. The variables must then be transformed to employ 
log-linearizations. In the case when $\gamma=1$, Christiano (1988) found such a transformation, but for $\gamma \neq 1$ there is no available transformation.

The value function $V(K, a)$ gives the expected discounted utility of having an amount of capital $K$ when the current productivity is $a$. As long as there are shocks to productivity, output and consumption will fluctuate. The counterfactual scenario in which there are no fluctuations in income corresponds to a world in which productivity does not vary but remains constant at $E\left[A_{t}\right]$.

While the costs of fluctuations are defined as before, now one must specify at which $(K, a)$ pair do they apply. The stationary steady state capital stock of the non-stochastic economy is a natural choice for $K$. As for $a$, I will calculate the expected costs of fluctuations by taking the expected value of $V(K, a)$ over the different possible realizations of $a$.

The appendix shows that if productivity is stationary, then:

$$
\ln (1+\lambda)=\left\{\begin{array}{l}
\frac{0.5 \omega^{2}}{1-\phi^{2}}\left[1-\left(1-e^{g-r}\right) V_{a a}\right]+O\left(\omega^{4}\right) \text { if } \gamma=1 \\
\frac{0.5 \omega^{2}}{1-\phi^{2}}+\frac{1}{\gamma-1} \ln \left[1+\frac{0.5 \omega^{2} V_{a a}}{\left(1-\phi^{2}\right) V}\right]+O\left(\omega^{4}\right) \quad \text { if } \gamma \neq 1
\end{array}\right.
$$

while if productivity is non-stationary and $\gamma=1$, then:

$$
\ln (1+\lambda)=0.5 \omega^{2}\left[\frac{1}{1-e^{g-r}}-\left(1-e^{g-r}\right)\left(V_{a a}-V_{a}\right)\right]+O\left(\omega^{4}\right) .
$$

Subscripts denote partial derivatives and all the functions are evaluated at the non-stochastic steady state; analytical expressions for each term are in the appendix. Given how small the $\omega$ 's typically are, the error in the expressions should be negligible.

Estimates of the costs of fluctuations in the economic model

Table 6 presents the estimated costs of fluctuations. Panel A has the estimates for the model with quickly diminishing returns to capital $(\alpha=0.36)$ and stationary productivity $(\phi=0.9)$. The costs of fluctuations are small, around $0.09 \%$ of consumption. While these are the smallest numbers in the table, they are already twice larger than the Lucas baseline.

In Panel B, productivity is still stationary, but there are only mildly diminishing returns to scale $(\alpha=0.75)$. Technology shocks now have a more long-lasting impact, and correspondingly the costs of fluctuations are two to three times larger. According to these estimates, each person in the United States would be willing to pay between $\$ 31$ and $\$ 55$ 
to eliminate fluctuations in consumption.

Table 6 - The costs of fluctuations in the stochastic growth model

\begin{tabular}{cccc}
\hline \hline \multicolumn{2}{c}{ Panel A: Stationary productivity and strongly diminishing returns } \\
& $\gamma=1$ & $\gamma=3$ & $\gamma=5$ \\
$r-g=0.03$ & $0.07 \%$ & $0.08 \%$ & $0.09 \%$ \\
& $(\$ 16)$ & $(\$ 19)$ & $(\$ 22)$ \\
$r-g=0.02$ & $0.07 \%$ & $0.09 \%$ & $0,10 \%$ \\
& $(\$ 17)$ & $(\$ 21)$ & $(\$ 24)$ \\
$r-g=0.01$ & $0.08 \%$ & $0.10 \%$ & $0.12 \%$ \\
& $(\$ 19)$ & $(\$ 24)$ & $(\$ 27)$ \\
\hline
\end{tabular}

Panel B: Stationary productivity and mildly diminishing returns

\begin{tabular}{cccc} 
& $\gamma=1$ & $\gamma=3$ & $\gamma=5$ \\
$r-g=0.03$ & $0.17 \%$ & $0.15 \%$ & $0.13 \%$ \\
$r-g=0.02$ & $(\$ 40)$ & $(\$ 36)$ & $(\$ 31)$ \\
& $0.18 \%$ & $0.18 \%$ & $0.16 \%$ \\
$r-g=0.01$ & $(\$ 43)$ & $(\$ 42)$ & $(\$ 38)$ \\
& $0.21 \%$ & $0.23 \%$ & $0.23 \%$ \\
\hline
\end{tabular}

Panel C: Non-stationary productivity

$\begin{array}{ccc} & \alpha=0.36 & \alpha=0.75 \\ r-g=0.03 & 0.55 \% & 0.26 \% \\ & (\$ 129) & (\$ 60) \\ r-g=0.02 & 0.85 \% & 0.39 \% \\ r-g=0.01 & (\$ 199) & (\$ 92) \\ & 1.76 \% & 0.81 \% \\ & (\$ 412) & (\$ 190)\end{array}$

Each cell shows the per capita costs of fluctuations as a fraction of consumption and, in brackets, in 2003 dollars.

Panel C finally turns to the case when productivity is non-stationary $(\phi=1)$. Consumption fluctuations are now much more costly. With quickly diminishing returns to scale, they cost between $0.6 \%$ and $1.8 \%$ of per capita consumption; slowly diminishing returns to scale lower these estimates by half. Increasing the speed of diminishing returns therefore raises the costs of fluctuations, the opposite of what happened with stationary productivity. The reason is that with non-stationary productivity, consumption is already a random walk in steady state, so that with mildly diminishing returns to scale, the model predicts 
that consumption is too persistent. Fitting the unconditional variance of first-differenced consumption predicted then requires a lower calibrated value for the volatility of shocks, which pushes the costs of fluctuations down.

The class of models analyzed in this section is just one among many different possibilities. Aside from generating estimates of the costs of fluctuations that are interesting in their own right, these models served a dual purpose. First, they showed how to calculate the costs of fluctuations within economic models that take the precautionary savings and investment risk effects into account. Second, they showed that the model's predicted persistence of consumption is a key determinant of the costs of fluctuations. This opens the door to estimating the costs of fluctuations in models that have other mechanisms propagating shocks over time aside from investment, such as for instance nominal rigidities or credit market frictions.

\section{Conclusion}

This paper re-examined the estimation of the costs of fluctuations, by focusing on the properties of aggregate consumption. It showed that the properties of the stochastic process describing consumption, and especially the persistence of shocks, are a key determinant of the costs of fluctuations. While the assumptions made by Lucas (1987) are decisively rejected by the data, this paper has shown that if one knows with certainty that shocks to consumption are only mildly persistent, the estimated costs of fluctuations are close to those that Lucas estimated.

The evidence though suggests that consumption fluctuations are more persistent than this. As persistence increases, the costs of fluctuations rise substantially. For instance, if consumption is a random walk, as some theories suggest and the data does not reject, the costs of fluctuations are fifty times larger than what Lucas estimated. The statistical models that best fit the data and the economic models that account for the effect of fluctuations on precautionary savings lead to even larger estimates of the costs of fluctuations, typically two orders of magnitude larger than Lucas' benchmark.

The conclusion that the costs of fluctuations are large and that they are driven mostly by persistent shocks was also suggested by Alvarez and Jermann (2004). This paper and theirs are very different however. First, they use asset pricing data to infer the marginal utility of 
consumption so they implicitly assign a large value for risk aversion, whereas I consider value for relative risk aversion of at most 5 . Second, they estimate the covariance of consumption with asset prices, whereas I estimate the persistence of consumption. Third, whereas they tackle the problem of estimating risk premia, I tackle the problem of estimating persistence when it is large. And fourth and finally, whereas they emphasize the need for a model of how consumers trade risk, I emphasize the need for a model of consumption dynamics over time. It is a demonstration of the power of the science of economics that sometimes, even if only rarely, we can pursue completely different measurement strategies and yet obtain some convergence in estimates and conclusions.

As the introduction discussed, it is unclear whether the fluctuations behind the estimates in this paper correspond to business cycles. If business cycles are transitory short-lived deviations of consumption away from a stable trend, as defined by for instance the use of band-pass filters, this paper suggests that the costs of business cycles are small. However, there is an alternative view of business cycles that dates back at least to Burns and Mitchell (1946) and which defines cycles as a set of regularities in the comovement of macroeconomic series. Campbell and Mankiw (1987) found that output fluctuations in the United States are actually very long-lived. In turn, Kydland and Prescott (1982) found that a calibrated real business cycle model driven by non-stationary productivity shocks fits quite closely the data on U.S. business cycles, and it predicts infinitely-lived consumption fluctuations. Under this view of business cycles, the welfare costs may be quite large. Whichever view one takes of business cycles, the calculations in this paper have at least provided the tools to estimate the costs of business cycles under different scenarios.

The bulk of the estimates in this paper suggest that the costs of fluctuations lie in the range from $0.5 \%$ to $5 \%$. These are significant amounts. To put these numbers into perspective, in 2003 the total amount spent by the U.S. federal government in unemployment and medical insurance was $\$ 53$ billions, or $0.8 \%$ of consumption; the amount spent in consumption by the federal government excluding national defense was $\$ 223$ billions (3.3\%); the amount spent in health coverage for low-income families through the Medicaid program was $\$ 265$ billions (3.9\%). ${ }^{16}$ The estimates in this paper suggest that eliminating fluctuations in consumption could be as valuable to society as the current protection against unemployment and the current provision of health care to the poor. If the federal government was

\footnotetext{
${ }^{16}$ Source: National Income and Product Accounts, tables 3.9.5 and 3.12.
} 
able to devise some policy that eliminated consumption fluctuations, consumers would be willing to reward it by almost doubling its non-defense budget.

These estimates still do not overturn the main Lucas (1987) point. Raising the economy's growth rate by $1 \%$ would have a much larger effect on welfare than eliminating fluctuations likely ever would. This comparison is only fair though insofar as it is as easy to raise a country's growth rate as it is to dampen fluctuations. There is little evidence that the recommendations of economists have had any effect on growth, let alone a substantial one (Easterly, 2002), but there is some evidence that advances in economic knowledge have led to policies that have stabilized the economy (Romer and Romer, 2002). A more fair comparison may be with other policies that seem within the scope of public policy. As Lucas (2003) discusses, lowering inflation from $10 \%$ to zero would imply a gain of $1 \%$ of consumption. Eliminating capital income taxes would raise per capita consumption by 2 to $4 \%$. The numbers in this paper put an upper bound on the benefits of eliminating fluctuations (short-run or not) that is in this range as well.

If an economist was able to come up with a policy that, when implemented, made a country grow $1 \%$ faster forever, his work would have a more important on society's welfare than probably any other economist has ever had. Until this happens though, lowering inflation, reducing taxes on capital income, and dampening consumption fluctuations, are aims that are within the grasp of our knowledge. If better stabilization policy can bring society a gain of $\$ 200$ billions, this is a large enough impact on well-being to motivate the work of a modest economist. 


\section{Appendix}

This appendix contains calculations omitted in the main text.

The costs of fluctuations in statistical models

For the case $\gamma=1$, the definition of the costs of fluctuations in (1) and of the counterfactual in statistical models imply that:

$$
\ln (1+\lambda)+\left(1-e^{-\rho}\right) E\left[\sum_{t=0}^{\infty} e^{-\rho t} E\left(c_{t}\right)\right]=\left(1-e^{-\rho}\right) \sum_{t=0}^{\infty} e^{-\rho t}\left(E\left(c_{t}\right)+0.5 \operatorname{Var}\left(c_{t}\right)\right)
$$

This result used the $\log$-normality of $C_{t}$ to evaluate $\ln \left(E\left(C_{t}\right)\right)$. Rearranging and substituting $\rho$ for $r-g$ gives the first expression in (2). For $\gamma \neq 1$, log-normality of consumption implies that $E\left(C_{t}^{1-\gamma}\right)=E\left(C_{t}\right)^{1-\gamma} e^{0.5 \gamma(\gamma-1) \operatorname{Var}\left(c_{t}\right)}$. Similar rearrangements lead to the second expression in (2).

The costs of fluctuations in the AR(1) statistical models

For a stationary $\operatorname{AR}(1), \operatorname{Var}\left(c_{t}\right)=\sigma^{2}\left(1-\eta^{2 t}\right) /\left(1-\eta^{2}\right)$ for $t \geq 1$. When $\gamma=1$, evaluating the sum in (2) shows that

$$
\ln (1+\lambda)=\frac{0.5 \sigma^{2}}{e^{r-g}-\eta^{2}}
$$

Using the approximations $e^{r-g}-1 \cong r-g$ and $\ln (1+\lambda) \cong \lambda$ gives the result.

For the case when $\gamma \neq 1$, approximate

$$
\ln (1+\lambda)=\frac{1}{\gamma-1} \ln \left[\left(1-e^{g-r}\right) \sum_{t=0}^{\infty} e^{(g-r) t} e^{0.5 \gamma(\gamma-1) \sigma^{2}\left(1-\eta^{2 t}\right) /\left(1-\eta^{2}\right)}\right]
$$

around $\sigma^{2}=0$ using a first-order Taylor expansion. Terms of order $\sigma^{4}$ or higher are tiny in the data, so this involves little error. This leads immediately to the same expression as in the $\log$ case, but now multiplied by $\gamma: \ln (1+\lambda) \cong 0.5 \gamma \sigma^{2} /\left(e^{r-g}-\eta^{2}\right)$. Similar approximations to before give the final result.

The costs of fluctuations in the benchmark economic model

The Euler equation for the problem in (6)-(7) is:

$$
C_{t}^{-\gamma}=e^{-\rho} E_{t}\left[R_{t+1} C_{t+1}^{-\gamma}\right]
$$


Then, guess that consumption is linear in wealth, $C_{t}=\pi R_{t} K_{t}$, with a coefficient $\pi$ to be determined. The budget constraint implies that:

$$
\frac{C_{t+1}}{C_{t}}=\frac{R_{t+1} K_{t+1}}{R_{t} K_{t}}=R_{t+1}(1-\pi)
$$

Using this result to replace for $C_{t+1} / C_{t}$ in the Euler equation and the fact that $R_{t+1}$ is log-normally distributed, (A5) becomes:

$$
\gamma \ln (1-\pi)=(1-\gamma) r-\rho+0.5 \gamma(\gamma-1) \sigma^{2}
$$

This expression does not depend on any state variable, which confirms the initial guess. Combining (A6) with (A5) and using the definition of $g$ gives the result in (8).

The costs of fluctuations for $\gamma \neq 1$ solve the equation:

$$
(1+\lambda)^{1-\gamma}\left(1-e^{g-r}\right)^{1-\gamma} \sum_{t=0}^{\infty} e^{\left[-\rho+(1-\gamma)\left(g-0.5 \gamma \sigma^{2}\right)\right] t}=\left(1-e^{g-r+0.5(1-\gamma) \sigma^{2}}\right)^{1-\gamma} \sum_{t=0}^{\infty} e^{\left[-\rho+(1-\gamma)\left(g+0.5(1-\gamma) \sigma^{2}\right)\right] t}
$$

Use the definition of $g$ in (8) to replace for $\rho$ and obtain:

$$
(1+\lambda)^{1-\gamma}\left(1-e^{g-r}\right)^{1-\gamma} \sum_{t=0}^{\infty} e^{(g-r) t}=\left(1-e^{g-r+0.5(1-\gamma) \sigma^{2}}\right)^{1-\gamma} \sum_{t=0}^{\infty} e^{\left.\left[g-r+0.5(1-\gamma) \sigma^{2}\right)\right] t}
$$

Evaluating the sums and taking logs shows that:

$$
\ln (1+\lambda)=\frac{\gamma}{\gamma-1} \ln \left(\frac{e^{r-g}-e^{-0.5(\gamma-1) \sigma^{2}}}{e^{r-g}-1}\right)
$$

The case when $\gamma=1$ follows along the same steps.

The costs of fluctuations in the Epstein-Zin-Weil model

It is easy so show (e.g., see Weil, 1990) that optimal consumption is in (8) but now with:

$$
\theta g=r-\rho+0.5(\theta-1) \gamma \sigma^{2}
$$

The expected discounted utility from setting optimal consumption equals:

$$
\frac{\left(R_{0} K_{0}\right)^{1-\gamma}\left(1-e^{-\rho}\right)^{(1-\gamma) /(1-\theta)}\left(1-e^{g-r}\right)^{-\theta(1-\gamma) /(1-\theta)}}{\left(1-e^{-\rho}\right)(1-\gamma)}-\frac{1}{\left(1-e^{-\rho}\right)(1-\gamma)}
$$


With the preferences in (9), without fluctuations, discounted utility equals

$$
\frac{\left(1-e^{-\rho}\right)^{(1-\gamma) /(1-\theta)}\left(R_{0} K_{0}\right)^{1-\gamma}\left(1-e^{g-r-(\theta-1) 0.5 \gamma \sigma^{2} / \theta}\right)^{-\theta(1-\gamma) /(1-\theta)}}{\left(1-e^{-\rho}\right)(1-\gamma)}-\frac{1}{\left(1-e^{-\rho}\right)(1-\gamma)}
$$

Given the definition of the costs of fluctuations, $(1+\lambda)^{1-\gamma}$ equals the ratio of the first terms in (A12) and (A11). After cancelling some terms and taking logs, this equals:

$$
\ln (1+\lambda)=\frac{\theta}{\theta-1} \ln \left(\frac{e^{r-g}-e^{-0.5(\theta-1) \gamma \sigma^{2} / \theta}}{e^{r-g}-1}\right)
$$

Finally, note that a linear approximation of the right-hand side of (A13) in $\sigma^{2}$ around zero is equal to a linear approximation of the right-hand side of (A9).

\section{Asymptotic distributions for the extended auto-regressive model}

The first extension is to include the $\gamma \neq 1$ cases. The simplest way to do this is to approximate the definition of the costs of fluctuations in (2) around the point $\sigma^{2}=0$. This shows that up to terms that are $O\left(\sigma^{4}\right)$ the costs of fluctuations with $\gamma \neq 1$ just equal $\gamma$ times the costs for the $\log$ utility case. In the data, the estimates of $\sigma$ are typically tiny so the $\sigma^{4}$ terms being ignored are quantitatively insignificant.

The second extension is to the Dickey-Fuller regression. One change is that now $\varrho=$ $1+(c / n)\left(1-\sum_{j=1}^{k} \psi_{j}\right)$. Another change is that the distribution of $\varrho$ is affected by the presence of the constant and the trend. Stock (1991) showed that:

$$
n(\hat{\varrho}-1) \Rightarrow\left(1-\sum_{j=1}^{k} \psi_{j}\right)\left[\left(\int_{0}^{1} J^{\tau}(s)^{2} d s\right)^{-1}\left(\int_{0}^{1} J^{\tau}(s) d W(s)\right)+c\right]
$$

where $J^{\tau}(s)=J(s)-\int_{0}^{1}(2-6 r) J(r) d r-s \int_{0}^{1}(12 r-6) J(r) d r$. The distribution of the estimate of the costs of fluctuations is otherwise similar to before.

The costs of fluctuations in the stochastic growth model with stationary productivity

The value function is defined as:

$$
V(K, a)=\max _{\left\{C_{t}\right\}}\left\{E\left[\sum_{t=0}^{\infty} e^{-\rho t} u\left(C_{t}\right)\right] \text { s.t. } K_{t+1}=e^{(1-\alpha) a_{t}} K_{t}^{\alpha}+(1-\delta) K_{t}-C_{t}\right\}
$$


Define $z \equiv(\mu-\phi \tau) /(1-\phi)$ and transform the variables using the relation $\left(\tilde{a}_{t}, \tilde{c}_{t}, \tilde{k}_{t}\right)=$ $\left(a_{t}-z-\tau t, c_{t}-z-\tau t, k_{t}-z-\tau t\right)$. Note that $\tilde{a}_{t}=\phi \tilde{a}_{t-1}+w_{t}$. The problem then becomes

$$
V(K, a)=\max \left\{E\left[\sum_{t=0}^{\infty} e^{-\rho t} u\left(\tilde{C}_{t} e^{z+\tau t}\right)\right] \text { s.t. } e^{\tau} \tilde{K}_{t+1}=e_{t}^{(1-\alpha) \tilde{a}_{t}} \tilde{K}_{t}^{\alpha}+(1-\delta) \tilde{K}_{t}-\tilde{C}_{t}\right\}
$$

Let $v(\tilde{K}, \tilde{a})=\max \left\{E\left[\sum_{t=0}^{\infty} e^{-[\rho-(1-\gamma) \tau] t} \tilde{u}\left(\tilde{C}_{t}\right)\right]\right.$ s.t. $\left.e^{\tau} \tilde{K}_{t+1}=e_{t}^{(1-\alpha) \tilde{a}_{t}} \tilde{K}_{t}^{\alpha}+(1-\delta) \tilde{K}_{t}-\tilde{C}_{t}\right\}$. The new utility function, $\tilde{u}($.$) , is a simple monotonic transformation of the utility function$ such that $\tilde{u}\left(C_{t}\right)=\left\{c_{t}\right.$ if $\gamma=1$, or $C_{t}^{1-\gamma} /(1-\gamma)$ if $\left.\gamma \neq 1\right\}$; it serves only the purpose of avoiding carrying one irrelevant additive term throughout. It then follows that

$$
V(K, a)=\left\{\begin{array}{lr}
v(\tilde{K}, \tilde{a})+\frac{1}{1-e^{-\rho}}\left(z+\frac{\tau}{e^{\rho}-1}\right) & \text { if } \gamma=1 \\
e^{z(1-\gamma)} v(\tilde{K}, \tilde{a})-\frac{1}{(1-\gamma)\left(1-e^{-\rho}\right)} & \text { if } \gamma \neq 1
\end{array}\right.
$$

In the counterfactual case where productivity equals $A_{t}^{*}=E\left[A_{t}\right]=\exp (z+\tau t+$ $0.5 \operatorname{Var}(a))$, consider instead the transformation $\left(\tilde{a}_{t}^{*}, \tilde{c}_{t}^{*}, \tilde{k}_{t}^{*}\right)=\left(a_{t}^{*}-z-\tau t-0.5 \operatorname{Var}(a)\right.$, $\left.c_{t}^{*}-z-\tau t-0.5 \operatorname{Var}(a), k_{t}^{*}-z-\tau t-0.5 \operatorname{Var}(a)\right)$. By taking the exact same steps as in the previous paragraph, you can see that the transformed value function is the same $v($. as in the original problem, for the case where $\tilde{a}=0$ and $w^{\prime}=0$. The value of being in an economy without fluctuations then is:

$$
V^{*}\left(K, a^{*}\right)=\left\{\begin{array}{l}
v^{*}(\tilde{K}, 0)+\frac{1}{1-e^{-\rho}}\left(z+\frac{\tau}{e^{\rho}-1}\right)+\frac{0.5 \operatorname{Var}(a)}{1-e^{-\rho}} \quad \text { if } \gamma=1 \\
e^{0.5 \operatorname{Var}(a)} e^{z(1-\gamma)} v^{*}(\tilde{K}, 0)-\frac{1}{(1-\gamma)\left(1-e^{-\rho}\right)} \text { if } \gamma \neq 1
\end{array}\right.
$$

I compute the costs of fluctuations at the steady state capital stock $K^{s s}$ and integrating over the possible values of $a$. The definition of the costs of fluctuations in (1) implies:

$$
\begin{gathered}
\ln (1+\lambda) /\left(1-e^{-\rho}\right)+E_{a}\left[V\left(K^{s s}, a\right)\right]=V^{*}\left(K^{s s}, a^{*}\right) \quad \text { if } \gamma=1 \\
(1+\lambda)^{1-\gamma} E_{a}\left[V\left(K^{s s}, a\right)\right]+\frac{(1+\lambda)^{1-\gamma}-1}{(1-\gamma)\left(1-e^{-\rho}\right)}=V^{*}\left(K^{s s}, a^{*}\right) \quad \text { if } \gamma \neq 1,
\end{gathered}
$$

where $E_{a}[$.$] is the expectations operator over the random variable a$. Using the expressions for the value functions in (A18) and (A19), this becomes:

$$
\begin{aligned}
& \ln (1+\lambda)=0.5 \operatorname{Var}(a)-\left(1-e^{-\rho}\right)\left(E_{a}\left[v\left(\tilde{K}^{s s}, \tilde{a}\right)\right]-v^{*}\left(\tilde{K}^{s s}, 0\right)\right) \quad \text { if } \gamma=1 \\
& \ln (1+\lambda)=0.5 \operatorname{Var}(a)-\frac{1}{\gamma-1} \ln \left[E_{a}\left[v\left(\tilde{K}^{s s}, \tilde{a}\right)\right] / v^{*}\left(\tilde{K}^{s s}, 0\right)\right] \quad \text { if } \gamma \neq 1
\end{aligned}
$$


Finally, a Taylor approximation of $v\left(\tilde{K}^{s s}, \tilde{a}\right)$ around the non-stochastic steady state is:

$$
v\left(\tilde{K}^{s s}, \tilde{a}\right)=v^{*}+v_{a} \tilde{a}+\frac{v_{a a} \tilde{a}^{2}}{2}+\frac{v_{a a} \tilde{a}^{3}}{6}+O\left(\tilde{a}^{4}\right),
$$

where $v_{a^{(n)}}=\partial^{(n)} v(.) / \partial \tilde{a}^{(n)}$ and all the functions are evaluated at the non-stochastic steady state. Integrating over $\tilde{a}$ and using the fact that it is mean-zero normally distributed:

$$
E_{a}\left[v\left(\tilde{K}^{s s}, \tilde{a}\right)\right]=v^{*}+0.5 v_{a a} \operatorname{Var}(\tilde{a})+O\left(\omega^{4}\right)
$$

Replacing this result into the expressions in (A21), using the fact that $\operatorname{Var}(\tilde{a})=\omega^{2} /(1-$ $\phi^{2}$ ), and noting that $V_{a}=v_{a}$ from (A18), gives the resulting expression for the costs of fluctuations in (22).

The costs of fluctuations in the stochastic growth model with non-stationary productivity

One appropriate transformation now is $\left(\tilde{c}_{t}, \tilde{k}_{t}\right)=\left(c_{t}-a_{t}, k_{t}-a_{t-1}\right)$ so that:

$V(K, a)=\max \left\{E\left[\sum_{t=0}^{\infty} e^{-\rho t} u\left(\tilde{C}_{t} A_{t}\right)\right]\right.$ s.t. $\left.\tilde{K}_{t+1}=\tilde{K}_{t}^{\alpha} e^{-\alpha\left(\mu+w_{t}\right)}+(1-\delta) \tilde{K}_{t} e^{-\left(\mu+w_{t}\right)}-\tilde{C}_{t}\right\}$

Define $v(\tilde{K}, w)$ as in the stationary case, and obtain:

$$
V(K, a)=v(\tilde{K}, w)+\sum_{t=0}^{\infty} e^{-\rho t} E_{0}\left[a_{t}\right]
$$

The stable economy is the one in which $A_{t}^{*}=E_{0}\left[A_{t}\right]=\exp \left(E_{0}\left(a_{t}\right)+0.5 \operatorname{Var}_{0}\left(a_{t}\right)\right)$. The corresponding transformation is $\left(\tilde{c}_{t}^{*}, \tilde{k}_{t}^{*}\right)=\left(c_{t}^{*}-a_{t}^{*}, k_{t}^{*}-a_{t-1}^{*}\right)$. This now leads to

$V^{*}\left(K, a^{*}\right)=\max \left\{E_{0}\left[\sum_{t=0}^{\infty} e^{-\rho t} u\left(\tilde{C}_{t} A_{t}^{*}\right)\right]\right.$ s.t. $\left.\tilde{K}_{t+1}=\tilde{K}_{t}^{\alpha} e^{-\alpha\left(\mu+0.5 \omega^{2}\right)}+(1-\delta) \tilde{K}_{t} e^{-\left(\mu+0.5 \omega^{2}\right)}-\tilde{C}_{t}\right\}$

The problem of the consumer in the stable economy then equals the problem of the consumer in the fluctuating economy when $w=0.5 \omega^{2}$, so

$$
V^{*}\left(K, a^{*}\right)=v^{*}\left(\tilde{K}, 0.5 \omega^{2}\right)+\sum_{t=0}^{\infty} e^{-\rho t} E\left[a_{t}^{*}\right]
$$

The costs of fluctuations are still given by the top expression in (A20), but the new 
expressions for the value functions with and without fluctuations now lead to:

$$
\ln (1+\lambda)=0.5 \omega^{2}\left(1-e^{-\rho}\right) \sum e^{-\rho t} t-\left(1-e^{-\rho}\right)\left(E_{w}\left[v\left(\tilde{K}^{s s}, w\right)\right]-v\left(\tilde{K}^{s s}, 0.5 \omega^{2}\right)\right)
$$

The approximation of $v\left(\tilde{K}^{s s}, w\right)$ is now around the point $w=0.5 \omega^{2}$. Integrating over $w$ leads to

$$
\ln (1+\lambda)=\frac{0.5 \omega^{2}}{e^{\rho}-1}+0.5 \omega^{2}\left(1-e^{-\rho}\right)\left(v_{w}-v_{w w}\right)+O\left(\omega^{4}\right)
$$

Finally, the relation linking $V($.$) and v($.$) shows that v_{w}=V_{a}-1 /\left(1-e^{-\rho}\right)$, and that $v_{w w}=V_{a a}$. Rearranging (A29) gives the expression in (23).

Calculating the derivatives of the value function in the stationary productivity case

Bellman's principle of optimality implies that the problem defined in $v(\tilde{k}, \tilde{a})$ has the following dynamic programming formulation:

$$
v(\tilde{K}, \tilde{a})=\max _{\tilde{k}^{\prime}}\left\{\tilde{u}\left(e^{(1-\alpha) \tilde{a}} \tilde{K}^{\alpha}+(1-\delta) \tilde{K}-e^{\tau} \tilde{K}^{\prime}\right)+e^{-\rho+(1-\gamma) \mu} E\left[v\left(\tilde{K}^{\prime}, \phi \tilde{a}+w^{\prime}\right)\right]\right\} .
$$

The optimality conditions are:

$$
\begin{aligned}
v_{k}(\tilde{K}, \tilde{a}) & =e^{-\rho-\gamma \tau} R(\tilde{K}, \tilde{a}) E_{t}\left[v_{k}\left(g(\tilde{K}, \tilde{a}), \phi \tilde{a}+w^{\prime}\right)\right] \\
v_{k}(\tilde{K}, \tilde{a}) & =R(\tilde{K}, \tilde{a}) u^{\prime}(\tilde{c}(\tilde{K}, \tilde{a})) \\
v_{a}(\tilde{K}, \tilde{a}) & =(1-\alpha) e^{(1-\alpha) \tilde{a}} \tilde{K}^{\alpha} u^{\prime}(c(\tilde{K}, \tilde{a}))+e^{\tau-r} \phi E_{t}\left[v_{a}\left(g(\tilde{K}, \tilde{a}), \phi \tilde{a}+w^{\prime}\right)\right] . \\
R(\tilde{K}, \tilde{a}) & \equiv \alpha e^{(1-\alpha) a} \tilde{K}^{\alpha-1}+1-\delta
\end{aligned}
$$

The first equation is the Euler equation, the second is the envelope theorem condition with respect to $\tilde{K}$, and the third is the envelope theorem condition with respect to $\tilde{a}$. The fourth equation defines an auxiliary function (that corresponds to the return on capital), which is useful to reduce the length of the expressions. The optimal choice of $\tilde{K}^{\prime}$ is given by a function $g(\tilde{K}, \tilde{a})$. Using the resource constraint, the optimal choice of consumption is $\tilde{c}(\tilde{K}, \tilde{a})=e^{(1-\alpha) \tilde{a}} \tilde{K}^{\alpha}+(1-\delta) \tilde{K}-g(\tilde{K}, \tilde{a}) e^{\tau}$.

In the non-stochastic steady state, $g\left(\tilde{K}^{s s}, 0\right)=\tilde{K}^{s s}, \tilde{a}=0$ and $w^{\prime}=0$. The set of equations above then returns the steady state values of $R, v_{k}, v_{a}$, and $\tilde{K}^{s s}$. (When a function is written without its argument, it is being evaluated at the non-stochastic steady 
state.) Note that $r \equiv \ln (R)=\rho+\gamma \tau$. The Bellman equation and the resource constraint in turn give the solutions for $v$ and $\tilde{C}^{s s}$. Finally, it is trivial to use the equation defining $R(\tilde{K}, \tilde{a})$ to obtain $R_{k}$ and $R_{a}$.

To construct the Taylor approximation, I perturb the system with respect to $(\tilde{k}, \tilde{a})$ around the point $\left(\tilde{k}^{s s}, 0\right)$. Perturbing the Euler equation with respect to $\tilde{k}$ and evaluating the functions at $\left(\tilde{k}^{s s}, 0\right)$ gives:

$$
v_{k k}=R^{-1} R_{k} v_{k}+v_{k k} g_{k}
$$

Rearranging, this gives an expression for $g_{k}$ in terms of only one unknown $v_{k k}$. Likewise perturbing the envelope theorem condition with respect to capital gives another equation for $g_{k}$ and $v_{k k}$. Using the expression from the perturbed Euler equation to replace for $g_{k}$ in the perturbed envelope theorem equation and rearranging gives a quadratic in $v_{k k}$ :

$$
v_{k k}^{2}-\left[R\left(R-e^{\tau}\right) u^{\prime \prime}\left(c^{s s}\right)+R_{k} u^{\prime}\left(c^{s s}\right)\right] v_{k k}-R_{k} u^{\prime \prime}\left(c^{s s}\right) v_{k} e^{\eta}=0
$$

Aside from $v_{k k}$, all the other elements in this equation are known. It is therefore trivial to solve this equation for $v_{k k}$, picking the negative solution, since the value function is concave.

Next perturb (A31) and (A32) with respect to $\tilde{a}$. This leads to the system:

$$
\begin{aligned}
v_{k a}(1-\phi) & =R^{-1} R_{a} v_{k}+v_{k k} g_{a} \\
v_{k a} & =R_{a} u^{\prime}\left(c^{s s}\right)+R u^{\prime \prime}\left(c^{s s}\right)\left[(1-\alpha)\left(\tilde{k}^{s s}\right)^{\alpha}-g_{a} e^{\tau}\right]
\end{aligned}
$$

These are two linear equations in two unknowns: $v_{k a}$ and $g_{a}$. It is easy to solve the system to obtain these two values. Finally, perturbing (A33) with respect to $\tilde{a}$ and rearranging gives a simple expression for $v_{a a}$ :

$v_{a a}=\frac{g_{a} e^{\tau-r} \phi v_{k a}+(1-\alpha)^{2}\left(\tilde{k}^{s s}\right)^{\alpha} u^{\prime}\left(c^{s s}\right)+(1-\alpha)\left(\tilde{k}^{s s}\right)^{\alpha} u^{\prime \prime}\left(c^{s s}\right)\left[(1-\alpha)\left(\tilde{k}^{s s}\right)^{\alpha}-g_{a} e^{\tau}\right]}{1-e^{\tau-r} \phi^{2}}$.

All of the terms in the right hand side of this expression are known, so this gives $v_{a a}$.

Calculating the derivatives of the value function in the non-stationary productivity case 
Bellman's principle of optimality now implies:

$$
v(\tilde{K}, w)=\max _{\tilde{k}^{\prime}}\left\{\ln \left[\tilde{K}^{\alpha} e^{-\alpha\left(\mu+w_{t}\right)}+(1-\delta) \tilde{K} e^{-\left(\mu+w_{t}\right)}-\tilde{K}^{\prime}\right]+e^{-\rho t} E\left[v\left(\tilde{K}^{\prime}, w^{\prime}\right)\right]\right\}
$$

The set of optimality conditions, in the same order as before is:

$$
\begin{aligned}
v_{k}(\tilde{K}, w) & =e^{-\rho} R(\tilde{K}, w) E_{t}\left[v_{k}\left(\tilde{K}^{\prime}, w^{\prime}\right)\right] \\
v_{k}(\tilde{K}, w) & =R(\tilde{K}, w) u^{\prime}(\tilde{c}(\tilde{K}, \tilde{a})) \\
v_{w}(\tilde{K}, w) & =-v_{k}(\tilde{K}, w) \\
R(\tilde{K}, w) & \equiv \alpha e^{-\alpha(\mu+w)} \tilde{K}^{\alpha-1}+(1-\delta) e^{-(\mu+w)}
\end{aligned}
$$

The optimal choice of $\tilde{K}^{\prime}$ is still denoted by $g(\tilde{K}, w)$, and the optimal consumption amount now equals $\tilde{c}(\tilde{K}, w)=e^{-\alpha(\mu+w)} \tilde{K}^{\alpha}+(1-\delta) \tilde{K} e^{-(\mu+w)}-g(\tilde{K}, w)$.

Evaluating this set of equations at the non-stochastic steady state, $\tilde{K}=\tilde{K}^{\prime}=\tilde{K}^{s s}$ and $w=\omega^{2} / 2$, gives the steady state values $R, v_{k}, v_{a}, \tilde{k}^{s s}$, and straightforward manipulations give $v, \tilde{c}^{s s}, R_{k}$ and $R_{a}$.

Perturbing (A41) and (A42) with respect to $\tilde{k}$ gives the same expressions as in the stationary case (though the expressions for $R$ and $\tilde{c}^{s s}$ are of course different). Again, these two equations give the solutions for $v_{k k}$ and $g_{k}$. Perturbing (A43) with respect to $\tilde{k}$ and with respect to $w$ gives the system of two equations: $v_{w k}=-v_{k}-\tilde{k}^{s s} v_{k k}$ and $v_{w w}=-\tilde{K}^{s s} v_{k w}$. Solving for $v_{w w}$ then gives the solution: $v_{w w}=\tilde{K}^{s s}\left(v_{k}+\tilde{K}^{s s} v_{k k}\right)$.

\section{References}

[1] Alvarez, Fernando and Urban J. Jermann (2004) "Using Asset Prices to Measure the Cost of Business Cycles," Journal of Political Economy, vol. 112, pp. 1223-1256.

[2] Arrow, Kenneth J. (1971) Essays in the Theory of Risk-Bearing, Amsterdam, North Holland.

[3] Atkeson, Andrew and Christopher Phelan (1994) "Reconsidering the Costs of Business Cycles with Incomplete Markets," NBER Macroeconomics Annual 1994, Cambridge, MA, MIT Press, pp. 187-207. 
[4] Barlevy, Gadi (2004) "The Cost of Business Cycles under Endogenous Growth," American Economic Review, vol. 94 (4), pp. 964-990.

[5] Barro, Robert J. and Xavier Sala-i-Martin (2003) Economic Growth, $2^{\text {nd }}$ edition, Cambridge, MIT Press.

[6] Beaudry, Paul and Carmen Pages (2001) "The Cost of Business Cycles and the Stabilization Value of Unemployment Insurance," European Economic Review, vol. 45, pp. $1545-1572$.

[7] Burns, Arthur F. and Wesley C. Mitchell (1946) Measuring Business Cycles, New York, National Bureau of Economic Research.

[8] Campbell, John and N. Gregory Mankiw (1987) "Are Output Fluctuations Transitory?" Quarterly Journal of Economics, vol. 102, pp. 857-880.

[9] Chetty, Raj (2005) "Labor Supply and Risk Aversion: A Calibration Theorem," UC Berkeley unpublished.

[10] Chetty, Raj and Adam Szeidl (2004) "Consumption Commitments and Asset Prices," Harvard University unpublished.

[11] Christiano, Lawrence J. (1988) “Why Does Inventory Investment Fluctuate so Much?" Journal of Monetary Economics, vol. 21, pp. 247-280.

[12] Cocco, João (2003) "Portfolio Choice in the Presence of Housing," London Business School, unpublished.

[13] Cochrane, John (1987) "How Big is the Random Walk in GNP?" Journal of Political Economy, vol. 96, pp. 893-920.

[14] Dickey, David A. and Wayne A. Fuller (1979) "Distribution of the Estimators for Autoregressive Time Series with a Unit Root," Journal of the American Statistical Association, vol. 74 (1), pp. 427-31.

[15] Dolmas, James (1998) "Risk Preferences and the Welfare Cost of Business Cycles," Review of Economic Dynamics, vol. 1 (3), pp. 646-676.

[16] Easterly, William (2002) The Elusive Quest for Growth, Cambridge, MIT Press. 
[17] Elliott, Graham, Thomas J. Rothenberg, and James H. Stock (1996) "Efficient Tests for an Autoregressive Unit Root," Econometrica, vol. 64 (4), pp. 813-836.

[18] Epaulard, Anne and Aude Pommeret (2003) "Recursive Utility, Endogenous Growth, and the Welfare Cost of Volatility," Review of Economic Dynamics, vol. 6 (3), pp. 672-684.

[19] Epstein, Larry G. and Stanley E. Zin (1989) "Substitution, Risk Aversion, and the Temporal Behavior of Consumption and Asset Returns: A Theoretical Framework," Econometrica, vol. 57 (4), pp. 937-969.

[20] Friend, Irwin and Marshall E. Blume (1975) "The Demand for Risky Assets," American Economic Review, vol. 65 (5), pp. 900-922.

[21] Fuhrer, Jeffrey C. (2000) "Habit Formation in Consumption and its Implications for Monetary Policy," American Economic Review, vol. 90 (3), pp. 367-390.

[22] Gabaix, Xavier and David Laibson (2003) "The 6D Bias and the Equity Premium Puzzle," NBER Macroeconomics Annual 2002, Cambridge, MIT Press, pp. 257-312.

[23] Hall, Robert E. (1978) "Stochastic Implications of the Life-Cycle Permanent Income Hypothesis: Theory and Evidence," Journal of Political Economy, vol. 86 (6), pp. 971-987.

[24] Imrohoroğlu, Ayse (1989) "The Cost of Business Cycles with Indivisibilities and Liquidity Constraints," Journal of Political Economy, vol. 97 (6), pp. 1364-1383.

[25] Kimball, Miles S. (1990) "Precautionary Saving in the Small and in the Large," Econometrica, vol. 58 (1), pp. 53-73.

[26] King, Robert G. and Sergio T. Rebelo (1993) "Transitional Dynamics and Economic Growth in the Neoclassical Growth Model," American Economic Review, vol. 83 (4), pp. 908-931.

[27] Knight, Frank H. (1944) "Diminishing Returns from Investment," Journal of Political Economy, vol. 52, pp. 26-47.

[28] Krebs, Tom (2003) "Growth and Welfare Effects of Business Cycles in Economies with Idiosyncratic Human Capital Risk," Review of Economic Dynamics, vol. 6, pp. 846-868. 
[29] Krebs, Tom (2004) "Job Displacement Risk and the Costs of Business Cycles," Brown University working paper.

[30] Krusell, Per and Anthony A. Smith, Jr. (1999) "On the Welfare Effects of Eliminating Business Cycles," Review of Economic Dynamics, vol. 2 (2), pp. 245-272.

[31] Kwiatkowski, Denis, Peter C.B. Phillips, Peter Schmidt, and Yongcheol Shin (1992) "Testing the Null Hypothesis of Stationarity Against the Alternative of a Unit Root: How Sure Are We that Economic Time Series Have a Unit Root?" Journal of Econometrics, vol. 54, pp. 159-178.

[32] Kydland, Finn E. and Edward C. Prescott (1982) "Time to Build and Aggregate Fluctuations," Econometrica, vol. 50 (6), pp. 1345-1370.

[33] Lucas, Robert E., Jr. (1987) Models of Business Cycles, New York, Basil Blackwell.

[34] Lucas, Robert E., Jr. (2003) "Macroeconomic Priorities," American Economic Review, vol. 93 (1), pp. 1-14.

[35] McGrattan, Ellen R. and Edward C. Prescott (2003) "Average Debt and Equity Returns: Puzzling?" American Economic Review Papers and Proceedings, vol. 93 (2), pp. 292-297.

[36] Mehra, Rajnish and Edward C. Prescott (1985) "The Equity Premium: A Puzzle," Journal of Monetary Economics, vol. 15, pp. 145-161.

[37] Ng, Serena and Pierre Perron (2001) "Lag Length Selection and the Construction of Unit Root Tests with Good Size and Power," Econometrica, vol. 69 (6), pp. 1519-1554.

[38] Obstfeld, Maurice (1994) "Evaluating Risky Consumption Paths: The Role of Intertemporal Substitutability," European Economic Review, vol. 38, pp. 1471-1486.

[39] Otrok, Christopher (2001) "On Measuring the Welfare Cost of Business Cycles," Journal of Monetary Economics, vol. 47, pp. 61-92.

[40] Parker, Jonathan A. and Bruce Preston (2004) "Precuationary Saving and Consumption Fluctuations," American Economic Review, forthcoming. 
[41] Phillips, Peter C. B. and Pierre Perron (1988) "Testing for a unit root in time series regression," Biometrika, vol. 75 (2), pp. 335-346.

[42] Phillips, Peter C. B. (1998) "Impulse Response and Forecast Error Variance Asymptotics in Nonstationary VARs," Journal of Econometrics, vol. 83, pp. 21-56.

[43] Poterba, James M. (1998) "The Rate of Return to Corporate Capital and Factor Shares: New Estimates Using Revised National Income Accounts and Capital Stock Data," Carnegie-Rochester Conference Series on Public Policy, vol. 48, pp. 211-246.

[44] Prescott, Edward C. (1986) "Theory Ahead of Business Cycle Measurement," FRB Minneapolis Quarterly Review, vol. 10 (4).

[45] Reis, Ricardo (2004) "Inattentive Consumers," NBER working paper 10883.

[46] Romer, Christina and David Romer (2002) "The Evolution of Economic Understanding and Postwar Stabilization Policy," in Rethinking Stabilization Policy, Federal Reserve Bank of Kansas City, pp. 11-78.

[47] Saks, Raven and Stephen Shore (2004) "Risk and Career Choices," Harvard University, unpublished.

[48] Stock, James H. (1991) "Confidence Intervals for the Largest Autoregressive Root in U.S. Macroeconomic Time Series," Journal of Monetary Economics, vol. 28, pp. 435459.

[49] Stock, James H. (1994) "Unit Roots, Structural Breaks and Trends," chapter 46 in Handbook of Econometrics, edited by R. Engle and D. McFadden, Amsterdam, Elsevier, pp. 2739-2841.

[50] Storesletten Kjetil, Chris I. Telmer, and Amir Yaron (2001) "The Welfare Costs of Business Cycles Revisited: Finite Lives and Cyclical Variation in Idiosyncratic Risk," European Economic Review, vol. 45 (7), pp. 1311-1339.

[51] Tallarini, Thomas D., Jr. (2000) "Risk-Sensitive Real Business Cycles," Journal of Monetary Economics, vol. 45 (3), pp. 507-532.

[52] Weil, Philippe (1990) "Nonexpected Utility in Macroeconomics," Quarterly Journal of Economics, vol. 105 (1), pp. 29-42. 